\title{
An Explicitly Solvable Nonlocal Eigenvalue Problem and the Stability of a Spike for a Sub-Diffusive Reaction-Diffusion System
}

\author{
Y. Nec ${ }^{1}$, M. J. Ward ${ }^{1 *}$ \\ ${ }^{1}$ Department of Mathematics, University of British Columbia \\ 1984 Mathematics Road, Vancouver, V6T1Z2, BC, Canada
}

\begin{abstract}
The stability of a one-spike solution to a general class of reaction-diffusion (RD) system with both regular and anomalous diffusion is analyzed. The method of matched asymptotic expansions is used to construct a one-spike equilibrium solution and to derive a nonlocal eigenvalue problem (NLEP) that determines the stability of this solution on an $\mathcal{O}(1)$ time-scale. For a particular sub-class of the reaction kinetics, it is shown that the discrete spectrum of this NLEP is determined in terms of the roots of certain simple transcendental equations that involve two key parameters related to the choice of the nonlinear kinetics. From a rigorous analysis of these transcendental equations by using a winding number approach and explicit calculations, sufficient conditions are given to predict the occurrence of Hopf bifurcations of the one-spike solution. Our analysis determines explicitly the number of possible Hopf bifurcation points as well as providing analytical formulae for them. The analysis is implemented for the shadow limit of the RD system defined on a finite domain and for a one-spike solution of the RD system on the infinite line. The theory is illustrated for two specific RD systems. Finally, in parameter ranges for which the Hopf bifurcation is unique, it is shown that the effect of sub-diffusion is to delay the onset of the Hopf bifurcation.
\end{abstract}

Keywords and phrases: matched asymptotic expansions, nonlocal eigenvalue problem, winding number, Hopf bifurcation, sub-diffusion

Mathematics Subject Classification: 35K57, 35B25, 35B35

\section{Introduction}

In the singularly perturbed limit, many two-component reaction-diffusion (RD) systems allow for the existence of steady-state, or time-dependent, spatially localized solutions. In this class of solutions, spike patterns are those where one or both of the solution components concentrate, or localize, at certain points in the domain. For the situation where only one of the two solution components is localized, the spikes are said to exhibit semistrong interactions. In this semi-strong interaction limit, over the past decade there have been many studies of the stability and dynamics of spike-type patterns in a one-dimensional domain for specific reaction-diffusion systems, including the Gierer-Meinhardt (GM) model (cf. [39], [12], [13], [5], [8], [14], [38], [37], [36], [31], [7]), the Gray-Scott (GS) model (cf. [3], [4], [20], [15], [31], [1]),

\footnotetext{
${ }^{*}$ Corresponding author. E-mail: ward@math.ubc.ca
} 
the Schnakenberg model (cf. [30],[9]), and more recently, the Brusselator model (cf. [34]), [35]) and a reaction-diffusion model of urban crime (cf. [17]).

In these studies, a wealth of different analytical techniques have been used such as the method of matched asymptotic expansions, Lyapanov-Schmidt reductions, geometric singular perturbation theory, the study of nonlocal eigenvalue problems, and renormalization methods. In a multi-dimensional domain, there is also a growing literature on the stability and dynamics of localized spots (see [2] and the references therein), but the results and available analytical techniques are to a large extent rather different in nature than for the 1-D case.

Although there is now much analytical theory for the existence, stability, and dynamics of spike patterns in specific RD systems in 1-D, much less is known about how the results can extend to more general classes of RD systems. In this direction, there have been a few studies on the slow dynamics of pulses in a class of RD systems (cf. [6], [28], [41]). However, to date, there has been no comprehensive study of the stability of the pulse solutions in these more general systems. The main technical challenge is that for the stability analysis one must rigorously analyze the discrete spectrum of the following class of nonlocal eigenvalue problems (NLEP) for $\Phi(y)$ on the infinite line $-\infty<y<\infty$ :

$$
L_{0} \Phi-\mathcal{C}(\lambda) a(w) \int_{-\infty}^{\infty} b(w) \Phi d y=\lambda \Phi, \quad-\infty<y<\infty ; \quad \Phi \rightarrow 0 \quad \text { as } \quad|y| \rightarrow \infty .
$$

Here $w(y)$ is the homoclinic of $w^{\prime \prime}-w+Q(w)=0$ for certain $Q(w)$ with $Q(0)=Q^{\prime}(0)=0, L_{0} \Phi=\Phi^{\prime \prime}-\Phi+$ $Q^{\prime}(w) \Phi$ is the linearized operator around $w, b(w)$ and $a(w)$ are nonlinear functions with $a(0)=b(0)=0$, and $\mathcal{C}(\lambda)$ is a transcendental function of $\lambda$. Since this NLEP is non-self-adjoint and non-local, it is very difficult to find sufficient conditions for which all discrete eigenvalues of $(1.1)$ satisfy $\operatorname{Re}(\lambda)<0$. For simple power nonlinearities where $Q(w)=w^{p}$ with $p \geq 2, a(w)=w^{m}$ with $m>0$, and $b(w)=w^{r}$ with $r>0$, there are some rigorous results for the spectrum of (1.1) (see the survey [40]), but the theory is intricate and still incomplete.

Within this context, the main goal of this paper is to characterize analytically the stability of a onespike solution to a class of reaction-diffusion systems with either regular or sub-diffusion for which the spectrum of the associated NLEP can be found explicitly. The class of reaction-diffusion systems with regular diffusion that we will consider is formulated as

$$
v_{t}=\varepsilon_{0}^{2} v_{x x}-v+g(u) v^{p}, \quad \tau u_{t}=u_{x x}+\left(u_{b}-u\right)+\frac{1}{\varepsilon_{0}} f(u) v^{r} .
$$

Here $\varepsilon_{0} \ll 1, u_{b}>0$ is a constant, $p \geq 2, r>1$, and the properties of $g(u)$ and $f(u)$ are given below. Since (1.2) is posed on an infinite domain, by scaling we can set the diffusivity of $u$ to unity (as we have done). The sub-diffusive counterpart of (1.2) is

$$
\partial_{t}^{\gamma} v=\varepsilon^{2 \gamma} v_{x x}-v+g(u) v^{p}, \quad \tau \partial_{t}^{\gamma} u=u_{x x}+\left(u_{b}-u\right)+\varepsilon^{-\gamma} f(u) v^{r},
$$

where the anomaly exponent $\gamma$ is on the range $0<\gamma<1$. In (1.3), the definition of the sub-diffusive operator as applied to a function $h(t)$ is (see [24])

$$
\frac{d^{\gamma}}{d t^{\gamma}} h(t) \equiv-\frac{1}{\Gamma(-\gamma)} \int_{0}^{t} \frac{h(t)-h(t-\zeta)}{\zeta^{\gamma+1}} d \zeta, \quad 0 \leqslant \gamma<1 .
$$

Sub-diffusion has been observed in nature and in particular in biological systems, where diffusion is often hindered due to crowding effects of the medium. For a survey of anomalous diffusion and fractional calculus see [27], [19], and [26]. In the study of the stability of spatially uniform equilibria of RD systems with anomalous diffusion, using both dynamical systems method and Fourier transform analysis, somewhat counter-intuitive stability characteristics have been reported (cf. [11], [29], [10], [23], [22], [33])). However, much less is known about the stability of localized structures in the presence of sub-diffusion. For the case of super-diffusion, the stability and dynamics of an interface with a piecewise linear kinetics 
has been studied in [21]. More closely related to this work is the recent study of the stability and dynamics of spike patterns for a sub-diffusive GM model (cf. [24]) where $g(u)=u^{-q}$ and $f(u)=u^{-s}$ in (1.3).

In this paper we show that the spectrum of the NLEP associated with (1.2) can be determined explicitly for the sub-range of exponents where $p=2 r-3$ with $r>2$. This parameter range was not observed in previous stability analyses (cf. [39], [5], [38], [37], [13], [36]). Recently, the specific case $p=r=3$ was observed and used in [17] to solve explicitly the NLEP associated with the stability of hot-spot patterns for an RD system of urban crime. For this new sub-range $p=2 r-3$ of exponents with $r>2$, we will derive an explicit transcendental equation for the discrete eigenvalues $\lambda$ that governs the stability of a one-spike solution for (1.2) on an $\mathcal{O}(1)$ time-scale. From a detailed analysis of the roots of this transcendental equation by graphical considerations, a winding number approach and analytical manipulations, we will obtain an explicit and rigorous stability theory for one-spike equilibrium solutions of (1.2) for general $f(u)$ and $g(u)$. It is then shown that this stability analysis is easily extended to incorporate the effect of sub-diffusion in (1.3).

We remark that for a one-spike solution to (1.2) on the infinite line, the translation-invariance mode $\lambda=0$ is always an eigenvalue in the spectrum of the linearization. This is the only "small" eigenvalue in the spectrum of the linearization. As such, our NLEP stability theory will completely determine the linearized stability properties of a one-spike equilibrium solution for (1.2) on the infinite line. However, in the more general context of one- or multi-spike patterns on a finite domain, a separate analysis is typically required to determine additional stability thresholds regarding the small eigenvalues of order $\mathcal{O}\left(\varepsilon_{0}^{2}\right)$ in the spectrum of the linearization. Explicit calculations of these small eigenvalues, and their associated stability thresholds, have been done for some specific RD systems such as the GM (cf. [13]), the GS (cf. [16]), and the Brusselator (cf. [34]) RD models. However, such results are not currently available for general RD systems. The exponent set restriction $p=2 r-3$ and $r>2$ in (1.2), which allows for an explicitly solvable NLEP, does not aid in the calculation of the small eigenvalues associated with a multi-spike pattern of (1.2) on a finite domain.

In previous studies for the GM RD model, either a functional-analytic approach coupled to a numerical discretization of a BVP (cf. [37]) or an approach based on numerical computations of certain complicated hypergeometric functions (cf. [8]) was used to determine the spectrum of the NLEP and the Hopf bifurcation threshold for $\tau$. To date, as discussed in $\S 6$ of [37], with the exception of the GM shadow problem (cf. [38]), there have been no rigorous results (without computer assistance) proving the existence of a unique Hopf bifurcation threshold for the GM model. For the specific case $p=2 r-3$ and $r>2$ of the GM model, for which the spectrum of the NLEP is explicitly available, our theory is able to provide rigorous results for the uniqueness of the Hopf bifurcation threshold. In addition, our results prove the uniqueness of the Hopf bifurcation threshold for the more general system (1.2) under certain conditions on $f$ and $g$.

The outline of this paper is as follows: In $\S 2$ we analyze the existence of a one-spike solution to (1.2) in the corresponding shadow limit where the RD system reduces to a PDE coupled to an ODE (see [38] for the shadow GM system). We then derive an NLEP that determines the stability of this solution on an $\mathcal{O}(1)$ time-scale. In contrast to the case of a one-spike solution on the infinite line where $\lambda=0$ is in the spectrum of the linearization, the shadow problem admits an exponentially small in $\epsilon_{0}$ eigenvalue not captured by NLEP theory. As such, our NLEP analysis only provides conditions to ensure the metastability of a one-spike solution of the shadow problem. In $\S 2.1$ we show that the discrete spectrum of the associated NLEP is explicitly available for the special case where $p=2 r-3$ and $r>2$. In $\S 2.2$ rigorous metastability and instability results are given, and an explicit formulae for the unique Hopf bifurcation threshold of $\tau$ is provided. The theory is illustrated for two specific RD systems in $\S 2.3$. In $\S 2.4$ it is shown how to easily extend these results to the case of sub-diffusion. In $\S 3$ we extend the analysis of $\S 2$ to consider the existence and stability of a one-spike solution to (1.2) on the infinite line. The stability analysis is undertaken for the case $p=2 r-3$ for which the NLEP is solvable. Rigorous and explicit results are given to predict the occurrence of Hopf bifurcations in terms of the bifurcation parameter $\tau$ for general $f(u)$ and $g(u)$. Finally a brief concluding discussion is given in $\S 4$. 


\section{NLEP Stability Theory for the Shadow System}

In this section, we consider the limiting system obtained by letting $D \rightarrow \infty$ in the following class of reaction-diffusion system defined on a finite domain:

$$
\begin{gathered}
v_{t}=\varepsilon_{0}^{2} v_{x x}-v+g(u) v^{p}, \quad|x| \leq 1 ; \quad v_{x}=0 \quad \text { on } \quad x= \pm 1 \\
\tau u_{t}=D u_{x x}+\left(u_{b}-u\right)+\frac{1}{\varepsilon_{0}} f(u) v^{r} ; \quad|x| \leq 1 ; \quad u_{x}=0 \quad \text { on } \quad x= \pm 1 .
\end{gathered}
$$

Without loss of generality we have taken the domain to have length two. The limiting system for $D \rightarrow \infty$, called the shadow system, is the nonlocal problem for $v=v(x, t)$ and $u=u(t)$ given by (see [38], [12] for the shadow GM system)

$$
\begin{gathered}
v_{t}=\varepsilon_{0}^{2} v_{x x}-v+g(u) v^{p}, \quad|x| \leq 1 ; \quad v_{x}=0 \quad \text { on } \quad x= \pm 1 \\
\tau \frac{d u}{d t}=-\left(u-u_{b}\right)+\frac{1}{2 \varepsilon_{0}} f(u) \int_{-1}^{1} v^{r} d x .
\end{gathered}
$$

The sub-diffusive counterpart of this shadow problem is

$$
\begin{gathered}
\partial_{t}^{\gamma} v=\varepsilon^{2 \gamma} v_{x x}-v+g(u) v^{p}, \quad|x| \leq 1 ; \quad v_{x}=0 \quad \text { on } \quad x= \pm 1 \\
\tau \frac{d^{\gamma} u}{d t^{\gamma}}=-\left(u-u_{b}\right)+\frac{1}{2 \varepsilon^{\gamma}} f(u) \int_{-1}^{1} v^{r} d x
\end{gathered}
$$

where the fractional time-derivatives are defined by (1.4).

In this section we study the metastability properties of a one-spike equilibrium solution centered at $x=0$ to $(2.2)$. The theory is then extended to treat the sub-diffusive shadow problem (2.3). It is readily shown that this equilibrium solution of (2.2) is given asymptotically for $\varepsilon_{0} \rightarrow 0$ by

$$
v_{e} \sim g_{0}^{-1 /(p-1)} w\left(x / \varepsilon_{0}\right), \quad u_{e} \sim U_{0},
$$

where $w(y)>0$ is the unique homoclinic solution of

$$
w^{\prime \prime}-w+w^{p}=0, \quad-\infty<y<\infty ; \quad w \rightarrow 0 \quad \text { as } \quad|y| \rightarrow \infty ; \quad w^{\prime}(0)=0, \quad w(0)>0,
$$

which is given explicitly by (cf. [5])

$$
w(y)=\left\{\left(\frac{p+1}{2}\right) \operatorname{sech}^{2}\left(\frac{(p-1)}{2} y\right)\right\}^{1 /(p-1)} .
$$

In addition, we readily obtain from the steady-state of $(2.2 \mathrm{~b})$ that $U_{0}$ satisfies the nonlinear algebraic equation

$$
U_{0}-u_{b}=\frac{f_{0} b_{r}}{2 g_{0}^{r /(p-1)}}, \quad f_{0} \equiv f\left(U_{0}\right), \quad g_{0} \equiv g\left(U_{0}\right), \quad b_{r} \equiv \int_{-\infty}^{\infty} w^{r} d y .
$$

Remark 2.1. The problem of determining the existence of a one-spike equilibrium solution to (2.2) is reduced to the problem of determining the solution structure to the nonlinear algebraic problem (2.6) for different functions $f(u)$ and $g(u)$. This problem may have multiple solutions, a unique solution, or no solution, depending on the range of parameters and the choices of the kinetics $f(u)$ and $g(u)$. Explicit examples of the theory are given below in $\S 2.3$.

To determine the metastability properties of this solution for the regular diffusion problem (2.2), we look for a localized eigenfunction in the form

$$
v=v_{e}+e^{\lambda t} \Phi\left(x / \varepsilon_{0}\right), \quad u=u_{e}+e^{\lambda t} \eta,
$$


where $\eta$ is a constant. By linearizing (2.2), we obtain that

$$
\begin{gathered}
L_{0} \Phi+\frac{g_{0}^{\prime}}{g_{0}^{p /(p-1)}} w^{p} \eta=\lambda \Phi, \quad-\infty<y<\infty ; \quad \Phi \rightarrow 0 \quad \text { as } \quad|y| \rightarrow \infty, \\
(1+\tau \lambda) \eta=\frac{1}{2 \varepsilon_{0}}\left[f_{0}^{\prime} \eta\left(\int_{-1}^{1} v_{e}^{r} d x\right)+r f_{0}\left(\int_{-1}^{1} v_{e}^{r-1} \Phi\left(x / \varepsilon_{0}\right) d x\right)\right],
\end{gathered}
$$

where $L_{0}$ is the local operator defined by

$$
L_{0} \Phi \equiv \Phi^{\prime \prime}-\Phi+p w^{p-1} \Phi .
$$

Next, we substitute $v_{e}$ from (2.4) into the two integrals in (2.7b), and then solve (2.7b) for $\eta$. Upon substituting the result for $\eta$ into (2.7a) we obtain, after re-expressing the result using (2.6), the following nonlocal eigenvalue problem (NLEP) governing $\mathcal{O}(1)$ time-scale instabilities of the shadow problem $(2.2)$.

Proposition 2.2. For $0<\varepsilon_{0} \ll 1$, the linearized metastability properties of the one-spike equilibrium solution of the shadow problem (2.2) is determined by the spectrum of the NLEP

$$
L_{0} \Phi-\chi w^{p}\left(\frac{\int_{-\infty}^{\infty} w^{r-1} \Phi d y}{\int_{-\infty}^{\infty} w^{r} d y}\right)=\lambda \Phi, \quad-\infty<y<\infty ; \quad \Phi \rightarrow 0 \quad \text { as } \quad|y| \rightarrow \infty .
$$

Here $L_{0}$ is the local operator, as defined in (2.8), and the multiplier $\chi$ of the nonlocal term is given by

$$
\chi=\frac{r g_{0}^{\prime}}{g_{0}}\left[\frac{f_{0}^{\prime}}{f_{0}}-\frac{(1+\tau \lambda)}{U_{0}-u_{b}}\right]^{-1}
$$

Remark 2.3. The continuous spectrum for (2.9a) consists of the portion $\lambda<-1$ of the negative real axis. A one-spike solution to the shadow problem (2.2) is metastable, i.e. is linearly stable on an $\mathcal{O}(1)$ time-scale, provided that all discrete eigenvalues of the NLEP $(2.9)$ satisfy $\operatorname{Re}(\lambda) \leq 0$. A rigorous analysis of the spectrum of the NLEP is very difficult owing to the fact that it is non-self-adjoint and that $\chi$ also depends on $\lambda$. Rigorous results for the spectrum of the NLEP associated with the GM model corresponding to $g(u)=u^{-q}$ and $f(u)=u^{-s}$ for specific ranges of the exponents $p$ and $r$ were given in [39], [38], [37], and [13]. However, to date, there are no rigorous results for the spectrum of (2.9) for arbitrary exponents $p>1$ and $r>1$. Although the NLEP determines $\mathcal{O}(1)$ time-scale instabilities, there is also the possibility of a very weak translational instability resulting from an exponentially small eigenvalue of the form $\lambda=\mathcal{O}\left(e^{-c / \varepsilon_{0}}\right)$, where $c>0$. This eigenvalue arises from the interaction of the tail of the spike with the boundaries of the domain (see [12] for an analysis of these instabilities for the shadow GM model). We will not consider these instabilities here. Thus, if all discrete eigenvalues of the NLEP $(2.9)$ satisfy $\operatorname{Re}(\lambda) \leq 0$, we say that the one-spike solution is stable on an $\mathcal{O}(1)$ time-scale, or equivalently, is metastable.

Next, we show that the NLEP in (2.9) is explicitly solvable when $p=2 r-3$ and $r>2$. This fact was not observed in the previous stability analyses. For this sub-range of exponents, we will derive a transcendental equation for the discrete eigenvalues of (2.9). The resulting equation is then readily analyzed to provide detailed metastability and instability results for a one-spike equilibrium solution of the shadow problem (2.2).

\subsection{An Explicitly Solvable NLEP Problem}

Lemma 2.4. (From [18]): Consider the local eigenvalue problem $L_{0} \phi_{l}=\nu \phi_{l}$ on $\mathbb{R}$ for $\phi_{l} \in \mathcal{H}^{1}(\mathbb{R})$. This problem admits the eigenvalues $\nu_{0}>0, \nu_{1}=0$, and $\nu_{j}<0$ for $j>1$. The eigenvalue $\nu_{0}$ is simple, and the corresponding eigenfunction $\phi_{l 0}$ has one sign. 
Thus, there is exactly one unstable eigenvalue $\nu_{0}>0$ for the infinite-line local eigenvalue problem. By solving this local eigenvalue problem in terms of certain hypergeometric functions, a more explicit result for the spectrum of $L_{0}$ was obtained in [5].

Lemma 2.5. (From [5]): Let $J=J(p)$ be a positive integer such that $J<(p+1) /(p-1) \leq J+1$. Then, for $\phi_{l} \in \mathcal{H}^{1}(\mathbb{R})$, the local eigenvalue problem $L_{0} \phi_{l}=\nu \phi_{l}$ on $\mathbb{R}$ has $J+1$ discrete eigenvalues given by

$$
\nu_{j}=\frac{1}{4}[(p+1)-j(p-1)]^{2}-1, \quad j=0, \ldots, J .
$$

The continuous spectrum of $L_{0}$ lies in the range $-\infty<\nu<-1$, with $\nu$ real.

This result is Proposition 5.6 of [5]. Notice that $\nu_{0}>0, \nu_{1}=0$, and $\nu_{j} \in(-1,0)$ for $2 \leq j \leq J$. However, when $p \geq 3$, then $J=1$, and there are no discrete eigenvalues in the interval $(-1,0)$.

For the special case where $p=2 r-3$ and $r>2$, we now show that $\phi_{l 0} \equiv w^{r-1}$ is the principal eigenfunction of $L_{0}$ associated with the unique positive eigenvalue $\nu_{0}=\beta \equiv r^{2}-2 r$.

Lemma 2.6. Let $w$ satisfy $w^{\prime \prime}-w+w^{p}=0$ with $p>1$, and let $L_{0}$ be the local operator $L_{0} \phi \equiv$ $\phi^{\prime \prime}-\phi+p w^{p-1} \phi$. Then, when $p=2 r-3$ and $r>2$, we have

$$
L_{0} w^{r-1}=\beta w^{r-1}, \quad \beta \equiv r^{2}-2 r>0 .
$$

Proof. We use $w^{\prime \prime}=w-w^{p}$ and $\left(w^{\prime}\right)^{2}=w^{2}-2 w^{p+1} /(p+1)$ to calculate

$$
\begin{aligned}
L_{0} w^{r-1} & =\left(w^{r-1}\right)^{\prime \prime}-w^{r-1}+p w^{p+r-2}, \\
& =(r-1)(r-2) w^{r-3}\left(w^{\prime}\right)^{2}+(r-1) w^{r-2} w^{\prime \prime}-w^{r-1}+p w^{p+r-2}, \\
& =(r-1)(r-2) w^{r-3}\left(w^{2}-\frac{2}{p+1} w^{p+1}\right)+(r-1) w^{r-2}\left(w-w^{p}\right)-w^{r-1}+p w^{p+r-2}, \\
& =w^{r-1}\left(r^{2}-2 r\right)+w^{r+p-2}\left(p-(r-1)-\frac{2(r-1)(r-2)}{p+1}\right) .
\end{aligned}
$$

Therefore, $L_{0} w^{r-1}=\left(r^{2}-2 r\right) w^{r-1}$ when the factor multiplying $w^{r+p-2}$ vanishes. This implies that $p(p+1)=(r-1)[2(r-2)+(p+1)]$, which can be factored as $(p-(2 r-3))(p-(1-r))=0$. Therefore, $p=2 r-3$ or $p=1-r$. Since $p>1$ is needed, the only relevant root is $p=2 r-3$ for $r>2$.

Remark 2.7. In the analysis of [17] of the stability of hot-spot patterns for a reaction-diffusion model of urban crime it was observed for $p=3$ that $L_{0} w^{2}=3 w^{2}$. This identity, which allowed for an explicit determination of the spectrum of an NLEP in [17], corresponds to the special case $r=3$ in Lemma 2.6. Therefore, our result in Lemma 2.6 is a generalization of this result of [17].

Remark 2.8. Since the derivation of the result in Lemma 2.6 relies on the determination of an explicit first integral of the ground-state problem $w^{\prime \prime}-w+w^{p}=0$, this result is essentially a 1-D result and cannot be extended to the study of radially symmetric homoclinic spots of $\Delta w-w+w^{p}=0$ in two spatial dimensions. However, the framework of Lemma 2.6 can be extended to treat non power-law nonlinearities. In particular, upon replacing $v^{p}$ and $v^{r}$ in (1.2) with more general functions $Q(v)$ and $h(v)$, the resulting NLEP is explicitly solvable provided that a certain differential relation between $Q(v)$ and $h(v)$ is satisfied. Details of this extension are given in [32].

By using the identity (2.11), we now show that the discrete spectrum of the NLEP in Proposition 2.2 can be found explicitly.

Lemma 2.9. Let $p=2 r-3$ and $r>2$, so that $p>1$, and consider the NLEP

$$
L_{0} \Phi-\chi w^{p} \frac{\int_{-\infty}^{\infty} w^{r-1} \Phi d y}{\int_{-\infty}^{\infty} w^{r} d y}=\lambda \Phi, \quad-\infty<y<\infty ; \quad \Phi \rightarrow 0, \quad|y| \rightarrow \infty,
$$


with $\chi=\chi(\lambda)$. Then, for eigenfunctions $\Phi$ for which $\int_{-\infty}^{\infty} w^{r-1} \Phi d y \neq 0$, the discrete eigenvalues must satisfy the transcendental equation

$$
\lambda=\beta-\left(\frac{r}{2}\right) \chi .
$$

The remaining eigenfunctions $\Phi$, for which $\int_{-\infty}^{\infty} w^{r-1} \Phi d y=0$, are simply the eigenfunctions of $L_{0}$ corresponding to the zero eigenvalue and any negative real eigenvalues.

Proof. To establish (2.13) we use Green's identity on $w^{r-1}$ and $\Phi$. Since $L_{0}$ is self-adjoint, we integrate by parts and use the decay at infinity to conclude that $\int_{-\infty}^{\infty}\left(w^{r-1} L_{0} \Phi-\Phi L_{0} w^{r-1}\right) d y=0$. From $(2.11)$ and (2.12) with $p=2 r-3$ we then calculate that

$$
0=\int_{-\infty}^{\infty}\left(w^{r-1} L_{0} \Phi-\Phi L_{0} w^{r-1}\right) d y=\left(\chi \frac{\int_{-\infty}^{\infty} w^{3 r-4} d y}{\int_{-\infty}^{\infty} w^{r} d y}+\lambda-\beta\right) \int_{-\infty}^{\infty} w^{r-1} \Phi d y .
$$

Therefore, for eigenfunctions for which $\int_{-\infty}^{\infty} w^{r-1} \Phi d y \neq 0$, we get

$$
\lambda=\beta-\chi \frac{\int_{-\infty}^{\infty} w^{3 r-4} d y}{\int_{-\infty}^{\infty} w^{r} d y} .
$$

To calculate the integral ratio in (2.14), we multiply $w^{\prime \prime}-w+w^{2 r-3}=0$ by $w^{r-1}$ and we multiply $L_{0} w^{r-1}=\beta w^{r-1}$ by $w$. Upon subtracting the resulting two expressions we obtain

$$
w\left(w^{r-1}\right)^{\prime \prime}-w^{r-1} w^{\prime \prime}+(2 r-4) w^{3 r-4}=\beta w^{r} .
$$

Upon integrating this expression over $-\infty<y<\infty$ and using $w \rightarrow 0$ as $|y| \rightarrow \infty$, we obtain that $(2 r-4) \int_{-\infty}^{\infty} w^{3 r-4} d y=\beta \int_{-\infty}^{\infty} w^{r} d y$. Therefore, since $\beta=r^{2}-2 r$, the integral ratio in (2.14) is simply $\int_{-\infty}^{\infty} w^{3 r-4} d y=\left(\int_{-\infty}^{\infty} w^{r} d y\right)(r / 2)$. This yields $(2.13)$.

Next, consider the eigenfunctions for which $\int_{-\infty}^{\infty} w^{r-1} \Phi d y=0$. From (2.12), the facts that $w^{r-1}$ is the unique and one-signed principal eigenfunction of $L_{0}$, and that any eigenfunctions of the selfadjoint operator $L_{0}$ must be orthogonal, it follows that these other eigenfunctions must belong to the set eigenfunctions of $L_{0}$ corresponding to the zero eigenvalue and any negative real eigenvalues of $L_{0}$.

This result shows that when $p=2 r-3$ and $r>2$, the zero eigenvalue and any negative real eigenvalues of $L_{0}$ remain at fixed locations in $\operatorname{Re}(\lambda) \leq 0$ independent of the multiplier $\chi$ of the nonlocal term. Therefore, to analyze whether (2.12) has any eigenvalues in $\operatorname{Re}(\lambda)>0$, we only need to analyze the roots of (2.13).

\subsection{NLEP Stability Theory for the Shadow Problem: Regular Diffusion}

For the explicitly solvable case of the NLEP where $p=2 r-3$ and $r>2$, we obtain that the discrete eigenvalues of the NLEP (2.9) are the roots of (2.13) where $\chi$ is given in (2.9b). By using the expressions (2.9b) for $\chi$ and (2.6) for $U_{0}$, we can write (2.13) more conveniently as follows:

Proposition 2.10. For $p=2 r-3$ and $r>2$, the discrete eigenvalues $\lambda$ of the NLEP in Proposition 2.2 are the roots of the transcendental equation

$$
2(1+\tau \lambda)=\mathcal{G}(\lambda) \equiv d_{0}-\frac{d_{1}}{\beta-\lambda},
$$

where $d_{0}$ and $d_{1}$ are defined by

$$
d_{0}=\frac{f_{0}^{\prime} b_{r}}{g_{0}^{r /(2 r-4)}}, \quad d_{1}=\left(\frac{r^{2} g_{0}^{\prime}}{2 g_{0}}\right)\left(\frac{f_{0} b_{r}}{g_{0}^{r /(2 r-4)}}\right) .
$$

Here $\beta=r^{2}-2 r>0$. 
Remark 2.11. In terms of $d_{0}$ and $d_{1}$, the multiplier $\chi$ of the NLEP in (2.9a) is

$$
\chi=\frac{2 d_{1}}{r\left[d_{0}-2(1+\tau \lambda)\right]} .
$$

Notice that for the parameter range where $d_{0}>2$, the multiplier $\chi$ of the NLEP is not analytic in $\operatorname{Re}(\lambda)>0$, but rather has a simple pole at $\lambda=\left(d_{0}-2\right) /(2 \tau)>0$.

By determining conditions for which $(2.15 \mathrm{a})$ has real positive roots, we readily obtain some simple sufficient conditions, valid for any $\tau \geq 0$, for the instability of the equilibrium spike solution. Below, we shall refer to the roots of $(2.15 \mathrm{a})$ and the eigenvalues of the NLEP in Proposition 2.2 interchangeably.

Proposition 2.12. Suppose that either

(I) $\quad d_{1}<0$ and $\mathcal{G}(0)<2 ; \quad$ or

$(I I) \quad d_{1}>0$ and $\mathcal{G}(0)>2 ; \quad$ or

(III) $\quad d_{1}>0$ and $d_{0}<2$.

Then, for any $\tau>0,(2.15 a)$ has at least one root with $\lambda>0$ and $\lambda$ real. Thus, the NLEP has at least one unstable real eigenvalue and the equilibrium spike solution for (2.2) is unstable.

Proof. The proof is immediate. For $(I)$, since $d_{1}<0$, we have that $\mathcal{G}^{\prime}(\lambda)>0$ for all $\lambda \neq \beta$. Then, since $2(1+\tau \lambda)>\mathcal{G}(\lambda)$ at $\lambda=0$, and $\mathcal{G}(\lambda) \rightarrow+\infty$ as $\lambda \rightarrow \beta^{-}$, by the intermediate value theorem (2.15a) must have a root in $0<\lambda<\beta$. For $(I I)$, since $d_{1}>0$ then $\mathcal{G}^{\prime}(\lambda)<0$ for all $\lambda \neq \beta$. Thus, if $\mathcal{G}(0)>2,(2.15 \mathrm{a})$ has a unique positive root in $0<\lambda<\beta$. For $(I I I)$, since $\mathcal{G}(0)<2$ and $\mathcal{G}^{\prime}(\lambda)<0$ on $0<\lambda<\beta$, there is no root to (2.15a) on $0<\lambda<\beta$. However, since $\mathcal{G}(\lambda) \rightarrow+\infty$ as $\lambda \rightarrow \beta^{+}$with $\mathcal{G}^{\prime}(\lambda)<0$, (2.15a) must have a positive root in $\lambda>\beta$ for any $\tau>0$.

The next result is for the case $d_{1}<0$ and $\mathcal{G}(0)>2$ for two different ranges of $d_{0}$.

Proposition 2.13. Suppose that $d_{1}<0, \mathcal{G}(0)>2$ and $d_{0}<2$. Then, when $\tau>\tau_{t}>0$, (2.15a) has exactly two real positive roots located on the sub-range $0<\lambda<\beta$, while for $0<\tau<\tau_{t}$ there are no positive roots to (2.15a) in $\lambda>0$. Here $\tau_{t}>0$ is given uniquely by

$$
\tau_{t} \equiv-\frac{d_{1}}{2\left(\beta-\lambda_{t}\right)^{2}}, \quad \lambda_{t} \equiv \beta+\frac{d_{1}}{2-d_{0}}\left(1-\sqrt{1+\frac{\left(2-d_{0}\right) \beta}{d_{1}}}\right)<\beta .
$$

Next, if $d_{1}<0$ and $d_{0}>2$, so that necessarily $\mathcal{G}(0)>2$, then (2.15a) has two roots in $\lambda>\beta$ when $0<\tau<\tau_{+}$, where

$$
\tau_{+} \equiv-\frac{d_{1}}{2\left(\beta-\lambda_{+}\right)^{2}}, \quad \lambda_{+} \equiv \beta+\frac{d_{1}}{2-d_{0}}\left(1+\sqrt{1+\frac{\left(2-d_{0}\right) \beta}{d_{1}}}\right)>\beta .
$$

For $\tau_{+}<\tau<\tau_{t}$, (2.15a) has no positive real roots. Finally, for $\tau>\tau_{t}$, (2.15a) has two real positive roots on $0<\lambda<\beta$.

Proof. The proof is a simple calculus exercise. We first let $\tau>0$ and suppose that $d_{1}<0, \mathcal{G}(0)>2$ and $d_{0}<2$. Since $d_{1}<0$, then $\mathcal{G}(\lambda)$ is an increasing and convex function of $\lambda$ on $0<\lambda<\beta$, with $\mathcal{G}(\lambda) \rightarrow+\infty$ as $\lambda \rightarrow \beta^{-}$. Since $2(1+\tau \lambda)<\mathcal{G}(\lambda)$ at $\lambda=0$ and $2(1+\tau \lambda)$ is monotone increasing in $\tau$ for each fixed $\lambda>0$, it follows that (2.15a) will have exactly two real roots in $0<\lambda<\beta$ for $\tau$ sufficiently large, and no real roots when $\tau$ is sufficiently small. The threshold value of $\tau$ occurs when $2(1+\tau \lambda)$ is tangent to $\mathcal{G}(\lambda)$. This condition for tangency is that

$$
2(1+\tau \lambda)=d_{0}-\frac{d_{1}}{\beta-\lambda}, \quad 2 \tau=-\frac{d_{1}}{(\beta-\lambda)^{2}},
$$


has a solution on $0<\lambda<\beta$. Since $\mathcal{G}(0)=d_{0}-d_{1} / \beta>2$, it is readily shown that $0<\lambda_{t}<\beta$. This yields (2.18). When $d_{0}<2$, we have $2(1+\tau \lambda)>\mathcal{G}(\lambda)$ for all $\lambda>\beta$, and hence (2.15a) has no real roots in $\lambda>\beta$. A graphical illustration of this result is shown in the left panel of Fig. 1.

Next, we let $\tau \geq 0$ and suppose that $d_{1}<0, \mathcal{G}(0)>2$ and $d_{0}>2$. For $\tau=0$ there is a root to (2.15a) located at $\lambda=\beta+d_{1} /\left(2-d_{0}\right)>\beta$. For $\tau>0$ sufficiently small, a new second real positive root to (2.15a) emerges from $\lambda=\infty$. It is readily shown that two roots merge into a double root on the interval $\beta<\lambda<\infty$ at the tangency point where $\tau=\tau_{+}$. At the second tangency point where $\tau=\tau_{t}>\tau_{+}$, two new real positive roots to (2.15a) on $0<\lambda<\beta$ emerge, and they persist for all $\tau$ with $\tau>\tau_{t}$. A graphical illustration of this result is shown in the right panel of Fig. 1.
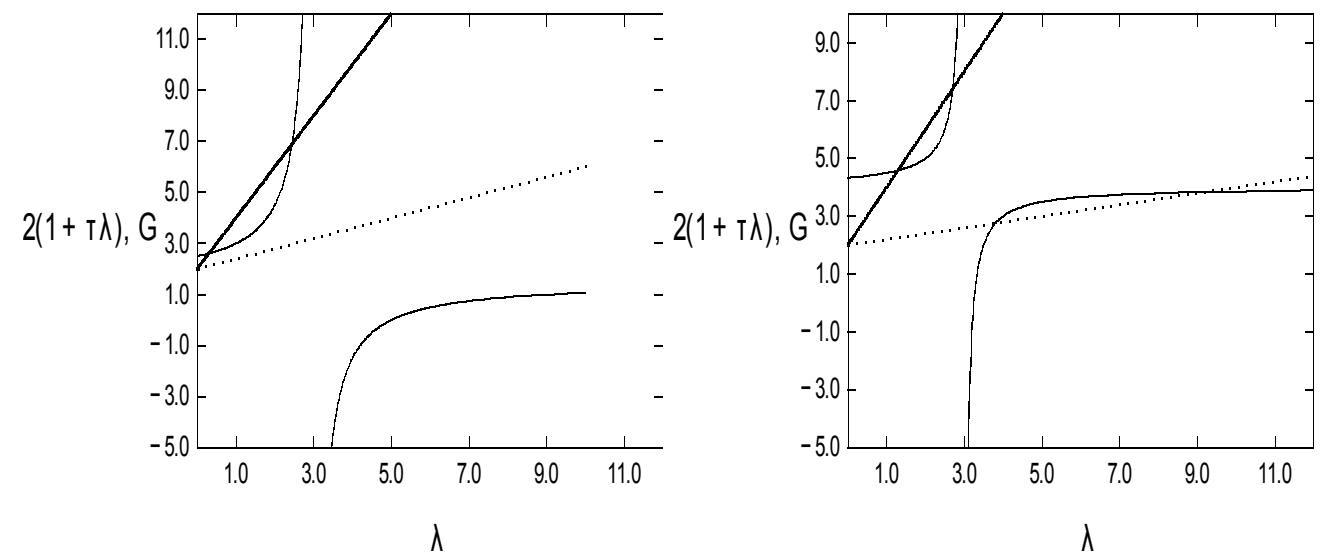

Figure 1. Left panel: Plot of $\mathcal{G}(\lambda)$ (solid curve) and $2(1+\tau \lambda)$ for $\tau=1.0$ (heavy solid line) and for $\tau=0.2$ (dotted line) versus $\lambda$ for $d_{0}=1.5, d_{1}=-3.0$, and $\beta=3.0$, for which $\mathcal{G}(0)=2.5>2$. There are two roots to $(2.15 \mathrm{a})$ when $\tau=1.0$ and none when $\tau=0.2$. Right panel: Plot of $\mathcal{G}(\lambda)$ (solid curve) and $2(1+\tau \lambda)$ for $\tau=1.0$ (heavy solid line) and for $\tau=0.1$ (dotted line) versus $\lambda$ for $d_{0}=4.0, d_{1}=-1.0$, and $\beta=3.0$, for which $\mathcal{G}(0) \approx 4.33>2$. There are two roots to $(2.15 \mathrm{a})$ when $\tau=1.0$ and when $\tau=0.1$.

Propositions 2.12 and 2.13 characterize the real positive roots of $(2.15 \mathrm{a})$, and suggest that a Hopf bifurcation may be possible for the case where $d_{1}<0, d_{0}<2$, and $\mathcal{G}(0)>2$. More specifically, Proposition 2.13 suggests that a complex conjugate pair of eigenvalues merges onto the positive real axis when $\tau=\tau_{t}>0$, raising the possibility that a Hopf bifurcation occurred for some $\tau=\tau_{H}>0$ with $\tau_{H}<\tau_{t}$.

To investigate this possibility, we first determine whether $(2.15 \mathrm{a})$ can have any pure imaginary roots. To this end, we set $\lambda=i \lambda_{I}$ and $\tau=\tau_{H}$ in (2.15a), where $\lambda_{I}>0$. Upon separating the real and imaginary parts of the resulting expression, we obtain a pure imaginary complex conjugate pair of eigenvalues when $\mathcal{G}(0)>2, d_{1}<0$ and $d_{0}<2$, given by

$$
\tau_{H}=\frac{\left(2-d_{0}\right)}{2 \beta}, \quad \lambda_{I}=\beta \sqrt{\frac{d_{1}}{\beta\left(d_{0}-2\right)}-1}=\beta \sqrt{\frac{\mathcal{G}(0)-2}{2-d_{0}}} .
$$

Notice that the extra condition $d_{0}<2$ is in fact needed. As $d_{0} \rightarrow 2^{-}$, then $\lambda_{I} \rightarrow+\infty$ and $\tau_{h} \rightarrow 0^{+}$. Therefore, in the case where $\mathcal{G}(0)>2, d_{1}<0$, and $d_{0}<2$, we have a pure imaginary complex conjugate pair of roots to $(2.15 \mathrm{a})$.

Next, we use a winding number criterion to establish a Hopf bifurcation result and to count precisely the number $N$ of roots of $(2.15 \mathrm{a})$ in $\operatorname{Re}(\lambda)>0$. We define $\mathcal{F}(\lambda) \equiv 2(1+\tau \lambda)-\mathcal{G}(\lambda)$, and note that 
$\mathcal{G}(\lambda)$ has a simple pole in $\operatorname{Re}(\lambda)>0$ at $\lambda=\beta$. Next, we calculate the winding number of $\mathcal{F}(\lambda)$ over the counterclockwise contour consisting of the imaginary axis $-i R \leq \operatorname{Im} \lambda \leq i R$ and the semi-circle $\Gamma_{R}$, given by $|\lambda|=R>0$, for $-\pi / 2 \leq \arg \lambda \leq \pi / 2$. For $\tau>0$, we have that $\mathcal{F}(\lambda) \sim 2 \tau \lambda$ as $|\lambda| \rightarrow \infty$ on $\Gamma_{R}$, so that the change in the argument of $\mathcal{F}$ over $\Gamma_{R}$ as $R \rightarrow \infty$ is $\pi$. By using the argument principle, together with $\mathcal{F}(\bar{\lambda})=\overline{\mathcal{F}(\lambda)}$, we obtain for $\tau>0$ that

$$
N=\frac{3}{2}+\frac{1}{\pi}[\arg \mathcal{F}]_{\Gamma_{I}},
$$

where $[\arg \mathcal{F}]_{\Gamma_{I}}$ denotes the change in the argument of $\mathcal{F}$ along the semi-infinite imaginary axis $\Gamma_{I}=i \lambda_{I}$, $0 \leq \lambda_{I}<\infty$, traversed in the downwards direction. From a direct calculation of (2.21) we obtain the following main result:

Proposition 2.14. Let $N$ denote the number of roots to (2.15a) in $\operatorname{Re}(\lambda)>0$. Suppose that $d_{1}<0$, $\mathcal{G}(0)>2$, and $d_{0}<2$. Then, $N=2$ for $\tau>\tau_{H}$, and $N=0$ for $\tau<\tau_{H}$, where $\tau_{H}$ is given in (2.20). Hence, the equilibrium spike solution for (2.2) undergoes a Hopf bifurcation at $\tau=\tau_{H}$. Alternatively, when $d_{1}<0, \mathcal{G}(0)>2$ but $d_{0}>2$, there is no Hopf bifurcation and $N=2$ for all $\tau>0$.

Proof. On $\Gamma_{I}$ we set $\lambda=i \lambda_{I}$ with $\lambda_{I}>0$. We use (2.15a) to separate the real and imaginary parts of $\mathcal{F}\left(i \lambda_{I}\right)$ as

$$
\mathcal{F}\left(i \lambda_{I}\right)=\mathcal{F}_{R}\left(\lambda_{I}\right)+i \mathcal{F}_{I}\left(\lambda_{I}\right), \quad \mathcal{F}_{R}\left(\lambda_{I}\right)=2-d_{0}+\frac{d_{1} \beta}{\beta^{2}+\lambda_{I}^{2}}, \quad \mathcal{F}_{I}\left(\lambda_{I}\right)=2 \lambda_{I}\left(\tau+\frac{d_{1}}{2\left(\beta^{2}+\lambda_{I}^{2}\right)}\right) .
$$

We first consider the case $d_{0}<2$. Then, for any $\tau>0$, we get $\mathcal{F}_{R}\left(\lambda_{I}\right) \rightarrow 2-d_{0}>0$ and $\mathcal{F}_{I}\left(\lambda_{I}\right) \rightarrow 2 \tau \lambda_{I}$ as $\lambda_{I} \rightarrow+\infty$ so that $\mathcal{F}_{I} / \mathcal{F}_{R} \rightarrow+\infty$ as $\lambda_{I} \rightarrow+\infty$. In addition, we calculate $\mathcal{F}_{R}(0)=2-d_{0}+d_{1} / \beta=$ $2-\mathcal{G}(0)<0$ and $\mathcal{F}_{I}(0)=0$. Moreover, for $d_{0}<2$, there is a unique point $\lambda_{I}^{*}$ in $0<\lambda_{I}<\infty$ where $\mathcal{F}_{R}\left(\lambda_{I}\right)=0$, given by $\lambda_{I}^{*}=\beta \sqrt{(\mathcal{G}(0)-2) /\left(2-d_{0}\right)}$. At this point we calculate $\mathcal{F}_{I}\left(\lambda_{I}^{*}\right)=2 \lambda_{I}^{*}\left(\tau-\tau_{H}\right)$, where $\tau_{H}$ is given in (2.20). Thus, $\mathcal{F}_{I}\left(\lambda_{I}^{*}\right)>0$ for $\tau>\tau_{H}$ and $\mathcal{F}_{I}\left(\lambda_{I}^{*}\right)<0$ if $\tau<\tau_{H}$. Consequently, $[\arg \mathcal{F}]_{\Gamma_{I}}=\pi / 2$ when $\tau>\tau_{H}$ and $[\arg \mathcal{F}]_{\Gamma_{I}}=-3 \pi / 2$ when $\tau<\tau_{H}$. Thus, from (2.21), we conclude that $N=0$ for $\tau<\tau_{H}$ and $N=2$ for $\tau>\tau_{H}$.

Next, we suppose that $d_{0}>2$. In this case, $\mathcal{F}_{R}\left(\lambda_{I}\right)<0$ for all $\lambda_{I}>0$ and $\mathcal{F}_{R}(0)=2-d_{0}+d_{1} / \beta<0$. Therefore, $[\arg \mathcal{F}]_{\Gamma_{I}}=\pi / 2$ for all $\tau>0$, and $(2.21)$ gives $N=2$ for all $\tau>0$.

Remark 2.15. For the range $d_{0}>2$ and $d_{1}<0$, it follows that $\mathcal{G}(0)>2$ by necessity. For this range, our results have proved that there are exactly two unstable eigenvalues in $\operatorname{Re}(\lambda)>0$ for any $\tau>0$. For $\tau>0$ and sufficiently small, there are two positive real roots to $(2.15 \mathrm{a})$ on the range $\lambda>\beta$, with one root tending to $+\infty$ while the other tending to $\lambda=\beta+d_{1} /\left(2-d_{0}\right)>\beta$ as $\tau \rightarrow 0^{+}$. For $\tau>0$ sufficiently large, there are two positive real roots to (2.15a) on the interval $0<\lambda<\beta$. For intermediate values of $\tau$, Proposition 2.14 proves that there is a pair of complex conjugate roots in $\operatorname{Re}(\lambda)>0$, and that the eigenvalues can never enter the stable left half-plane $\operatorname{Re}(\lambda)<0$. We recall from $(2.16)$ that for the range where $d_{0}>2$, the multiplier $\chi$ of the NLEP has a simple pole in $\operatorname{Re}(\lambda)>0$ at $\lambda=\left(d_{0}-2\right) /(2 \tau)>0$.

\subsection{Two Examples of the Theory}

We now illustrate our main results for two specific RD systems.

GM Model: We first consider the GM model where $g(u)=u^{-q}, f(u)=u^{-s}$ and $u_{b}=0$. Then, from the definitions of $d_{0}$ and $d_{1}$ in $(2.15 \mathrm{~b})$, we have

$$
d_{0}=-2 s, \quad d_{1}=-q r^{2}, \quad \beta=r^{2}-2 r .
$$

We remark that the condition $\mathcal{G}(0)>2$ is equivalent to the usual assumption (cf. [13]) on the GM exponent sets $(p, q, r, s)$ given by

$$
\frac{q r}{p-1}-(s+1)>0 \quad \text { where } \quad p=2 r-3 .
$$


From (2.6) we conclude that there is a unique one-spike solution and that $U_{0}^{\zeta}=2 / b_{r}$, where $\zeta \equiv$ $q r /(2 r-4)-(s+1)$.

We first suppose that $q<0$ so that $d_{1}>0$. Then, (II) and (III) of Proposition 2.12 hold, and hence the equilibrium spike solution is unstable for all $\tau>0$ due to a positive real eigenvalue. From (I) of Proposition 2.12, a similar instability occurs for $q>0$ but $q r /(2 r-4)-(s+1)<0$, so that $\mathcal{G}(0)<2$.

Next, suppose that $q>0, s>-1$ and that the usual assumption (2.24) holds on the GM exponent set $(p, q, r, s)$. Then, $d_{0}<2, d_{1}<0$, and $\mathcal{G}(0)>2$, so that Proposition 2.14 and (2.20) proves that an equilibrium spike solution to the GM shadow problem with $p=2 r-3$ has a Hopf bifurcation when $\tau=\tau_{H}$ with $\lambda=i \lambda_{I}$, where

$$
\tau_{H}=\frac{1+s}{r(r-2)}, \quad \lambda_{I}=r(r-2) \sqrt{\frac{q r}{2(1+s)(r-2)}-1} .
$$

Since $N=2$ for $\tau>\tau_{H}$, we conclude that for $\tau_{H}<\tau<\tau_{t}$ there is a pair of complex conjugate eigenvalues in $\operatorname{Re}(\lambda)>0$ that merge onto the positive real axis at $\lambda=\lambda_{t}$ when $\tau=\tau_{t}$. From (2.18) of Proposition 2.13 , we calculate

$$
\tau_{t}=\frac{q r^{2}}{2\left(r^{2}-2 r-\lambda_{t}\right)^{2}}, \quad \lambda_{t}=\left(r^{2}-2 r\right)-\frac{q r^{2}}{2(1+s)}\left(1-\sqrt{1-\frac{2(1+s)(r-2)}{q r}}\right) .
$$

For $\tau>\tau_{t}$, Proposition 2.13 shows that there are two real eigenvalues in $0<\lambda<\beta$.
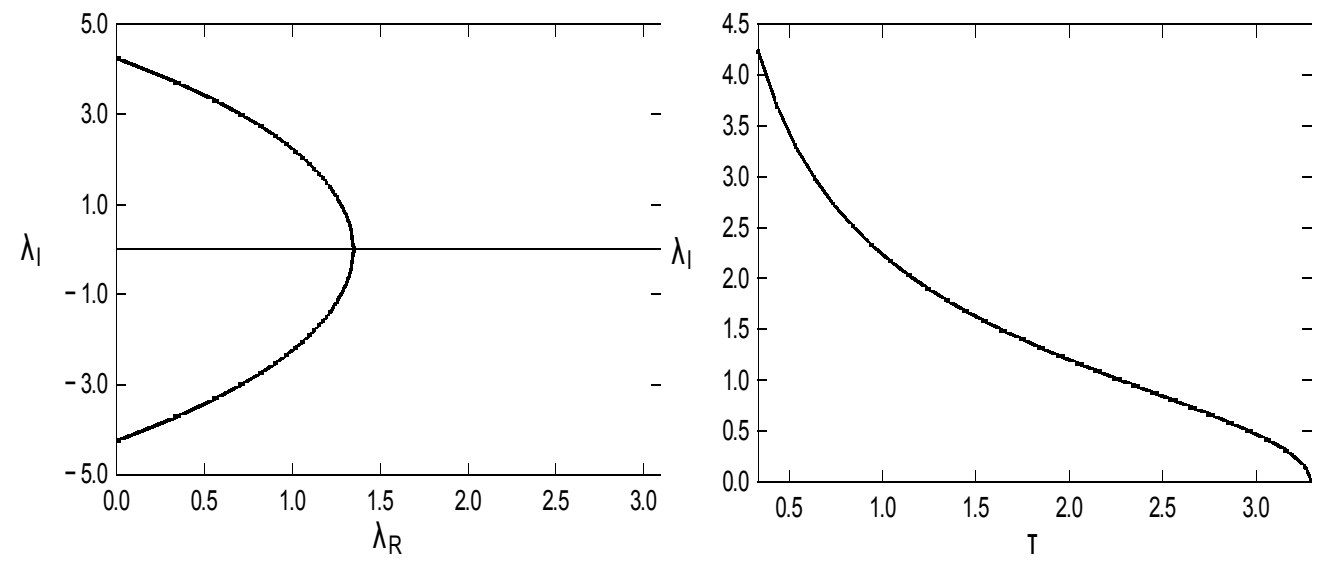

Figure 2. Plot of the complex conjugate pair of roots of (2.15a) in the complex $\lambda$-plane for the GM model with exponent set $(p, q, r, s)=(3,2,3,0)$. Left panel: Plot of $\operatorname{Im}(\lambda)$ versus $\operatorname{Re}(\lambda)$ for $\tau$ on the range $\tau_{H} \leq \tau \leq \tau_{t}$. Right panel: plot of $\operatorname{Im}(\lambda)>0$ versus $\tau$ on the range $\tau_{t} \leq \tau \leq \tau_{H}$. The endpoints of this curve are given analytically by (2.27).

For the particular exponent set $(p, q, r, s)=(3,2,3,0)$, we calculate from $(2.25)$ and (2.26) that

$$
\tau_{H}=1 / 3, \quad \lambda_{I H}=3 \sqrt{2} \approx 4.242, \quad \lambda_{t}=3-9\left(1-\sqrt{\frac{2}{3}}\right) \approx 1.348, \quad \tau_{t}=\frac{9}{\left(3-\lambda_{t}\right)^{2}} \approx 3.300 .
$$

For this exponent set, in Fig. 2 we plot the numerically computed path of the roots of $(2.15 \mathrm{a})$ in the complex $\lambda$-plane on the range $\tau_{H} \leq \tau \leq \tau_{t}$, and we also plot $\operatorname{Im}(\lambda)$ versus $\tau$ on this range. The results in this figure are seen to agree with (2.27). 
As a partial confirmation of our analytical theory, we next consider the exponent set $(p, q, r, s)=$ $(3,2,3,1)$ for which numerical results for the Hopf bifurcation threshold, as computed from a discretization of the associated NLEP, were given in the fourth row of Table 3 of [38]. From (2.25) and (2.26), our analytical theory predicts that

$$
\tau_{H}=2 / 3, \quad \lambda_{I H}=\frac{3 \sqrt{2}}{2} \approx 2.121, \quad \lambda_{t}=\frac{3(\sqrt{3}-1)}{3} \approx 1.098, \quad \tau_{t}=\frac{9}{\left(3-\lambda_{t}\right)^{2}} \approx 2.488 .
$$

The numerical results given in the fourth row of Table 3 of [38] closely agree with these values. To further validate our theory, we numerically solved the full PDE (2.2) for the GM model by using the numerical method described in [38]. The initial condition for (2.2) was taken to be a $1 \%$ perturbation of the quasi-equilibrium solution, so that $v(x, 0)=U_{0} w\left(x / \varepsilon_{0}\right)(1+0.01)$ and $u(0)=U_{0}(1+0.01)$, where $U_{0}=2 / b_{3}$. Here $w(y)=\sqrt{2} \operatorname{sech}(y)$ and $U_{0}=2 / b_{3}$, with $b_{3}=\sqrt{2} \pi$. For $\varepsilon_{0}=0.02$, in Fig. 3 we plot the numerically computed spike amplitude $v_{\mathrm{m}}(t)$, defined by $v_{\mathrm{m}}=v(0, t)$, versus $t$ for $\tau=0.64$ (dotted curve) and for $\tau=0.72$ (solid curve). For the smaller value of $\tau$, the amplitude oscillation decays, whereas an oscillatory instability occurs for the larger value of $\tau$. For $\tau=0.72$, the numerical results yield that the period of the oscillation is approximately 0.32 . In comparison, the theoretical result $(2.28)$ gives a Hopf bifurcation threshold of $\tau_{H}=2 / 3$, while the period of small-scale oscillations is $2 \pi / \lambda_{I H} \approx 0.29$.

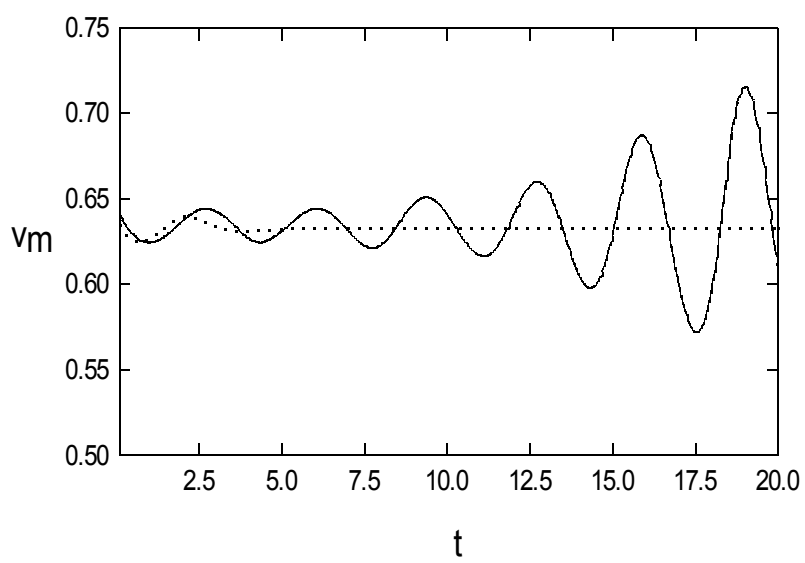

Figure 3. Plot of the spike amplitude $v_{\mathrm{m}}(0)=v(0, t)$ versus $t$ as numerically computed from the shadow PDE (2.2) with $\varepsilon_{0}=0.02$ for a GM model corresponding to setting $p=r=3, g(u)=u^{-2}, f(u)=u^{-1}$ and $u_{b}=0$ in (2.2). The dotted curve is for $\tau=0.64$, while the solid curve is for $\tau=0.72$. The theoretical prediction for the Hopf bifurcation threshold is $\tau_{H}=2 / 3$, which is between these two values.

Generalized GS Model: Next, we consider the shadow limit $D \rightarrow+\infty$ for a generalization of the GS model formulated as

$$
v_{t}=\varepsilon_{0}^{2} v_{x x}-v+\mathcal{A} u v^{3}, \quad \tau u_{t}=D u_{x x}-(u-1)-\frac{1}{\varepsilon_{0}} u v^{3} .
$$

on $|x|<1$. Here $\mathcal{A}>0$ is a parameter. This corresponds to setting

$$
p=r=3, \quad f(u)=-u, \quad g(u)=\mathcal{A} u, \quad u_{b}=1,
$$

in the shadow problem (2.2). This model is a generalization of the usual GS model with $p=r=2$, where pulse solutions have been analyzed in [3], [4], [20], [15], [31], and [1] (see also the references therein). 
For this model, the nonlinear algebraic equation (2.6) for $U_{0}$ reduces to

$$
\mathcal{H}\left(U_{0}\right) \equiv \sqrt{U_{0}}\left(1-U_{0}\right)=\frac{b_{3}}{2 \mathcal{A}^{3 / 2}}, \quad \text { where } \quad b_{3} \equiv \int_{-\infty}^{\infty} w^{3} d y=\sqrt{2} \pi,
$$

and $w=\sqrt{2} \operatorname{sech}(y)$ is the homoclinic of $w^{\prime \prime}-w+w^{3}=0$. Since $\mathcal{A}>0$, we must have $0<U_{0}<1$. In addition, since $\mathcal{H}\left(U_{0}\right)$ attains it maximum value on $0<U_{0}<1$ of $2 /(3 \sqrt{3})$ when $U_{0}=1 / 3$, it follows that the graph of $U_{0}$ versus $\mathcal{A}$ has a saddle-node bifurcation structure, with two solutions $U_{0 \pm}$ for $U_{0}$ when $\mathcal{A}>\mathcal{A}_{m}$, which satisfy $0<U_{0-}<1 / 3<U_{0+}<1$. Here, $A_{m}$ is given by

$$
\mathcal{A}_{m}=\left(\frac{3 \sqrt{6} \pi}{4}\right)^{2 / 3}
$$

A plot of the bifurcation diagram of $U_{0}$ versus $\mathcal{A}$ is shown in Fig. 4. In terms of $U_{0 \pm}$, the amplitude $v_{ \pm}(0)$ of the spike, as obtained from $(2.4)$, is

$$
v_{ \pm}(0)=\frac{w(0)}{\sqrt{\mathcal{A} U_{0 \pm}}}, \quad w(0)=\sqrt{2} .
$$

We refer to the root $U_{0-}$ on $0<U_{0-}<1 / 3$ as generating the "large" amplitude spike, while $U_{0+}$ on $1 / 3<U_{0+}<1$ generates the small amplitude spike. This classification follows from (2.33) since $v_{-}(0)>v_{+}(0)$. In summary, there are two one-spike equilibrium solutions when $\mathcal{A}>\mathcal{A}_{m}$, and none when $0<\mathcal{A}<\mathcal{A}_{m}$.

To determine the metastability properties of these solutions, we first identify that $\beta=r^{2}-3 r=3$ and then we calculate $d_{0}$ and $d_{1}$ from $(2.15 \mathrm{~b})$ as

$$
d_{0}=-\frac{b_{3}}{\left[\mathcal{A} U_{0}\right]^{3 / 2}}=-\frac{2\left(1-U_{0}\right)}{U_{0}}<0, \quad d_{1}=-\frac{9 b_{3}}{2\left[\mathcal{A} U_{0}\right]^{3 / 2}}=-\frac{9\left(1-U_{0}\right)}{U_{0}}<0, \quad \mathcal{G}(0)=\frac{\left(1-U_{0}\right)}{U_{0}} .
$$

Since $\mathcal{G}(0)=\left(1-U_{0}\right) / U_{0}<2$ when $U_{0}>1 / 3$, we conclude from (I) of Proposition 2.12 that the entire upper $U_{0+}$ branch of Fig. 4 is unstable due to a positive real eigenvalue for any $\tau>0$. Consequently, the small amplitude spike for $v$ is unstable for all $\tau>0$.

In contrast, along the lower $U_{0-}$ branch of Fig. 4 where $0<U_{0}<1 / 3$, we have $\mathcal{G}(0)>2, d_{0}<2$ and $d_{1}<0$. Therefore, from Propositions 2.13 and 2.14 we conclude that the one-spike solution is stable on an $\mathcal{O}(1)$ time-scale when $0<\tau<\tau_{H}$, and that there is a Hopf bifurcation at $\tau=\tau_{H}$ and $\lambda=i \lambda_{I}$. From (2.20) we obtain

$$
\tau_{H}=\frac{\left(2-d_{0}\right)}{2 \beta}=\frac{1}{3 U_{0}}, \quad \lambda_{I}=3 \sqrt{\frac{\left(1-3 U_{0}\right)}{2}} .
$$

For the range of $\tau$ where $\tau_{H}<\tau<\tau_{t}$, there is a complex conjugate pair of eigenvalues in $\operatorname{Re}(\lambda)>0$. These eigenvalues merge onto the real axis at $\lambda=\lambda_{t}$ when $\tau=\tau_{t}$. From (2.18), we obtain that

$$
\lambda_{t}=3-\frac{9}{2}\left(1-U_{0}\right)\left(1-\sqrt{\frac{1-3 U_{0}}{3\left(1-U_{0}\right)}}\right), \quad \tau_{t}=\frac{9\left(1-U_{0}\right)}{2 U_{0}\left(3-\lambda_{t}\right)^{2}} .
$$

In the left panel of Fig. 5 we use $(2.35 \mathrm{a})$ and $(2.35 \mathrm{~b})$ to plot $\tau_{H}$ and $\tau_{t}$ versus $U_{0}$ on the large amplitude spike branch $0<U_{0}<1 / 3$ of Fig. 4 . Then, by using (2.31) which relates $U_{0}$ to $\mathcal{A}$, in the right panel of Fig. 5 we correspondingly plot $\tau_{H}$ and $\tau_{t}$ versus $\mathcal{A}$ on this solution branch.

\subsection{NLEP Stability Theory with Sub-Diffusion}

In this subsection we study the metastability of a one-spike solution to the sub-diffusive shadow system (2.3). In the context of fractional differential equations, perturbations of a steady-state solution do not 


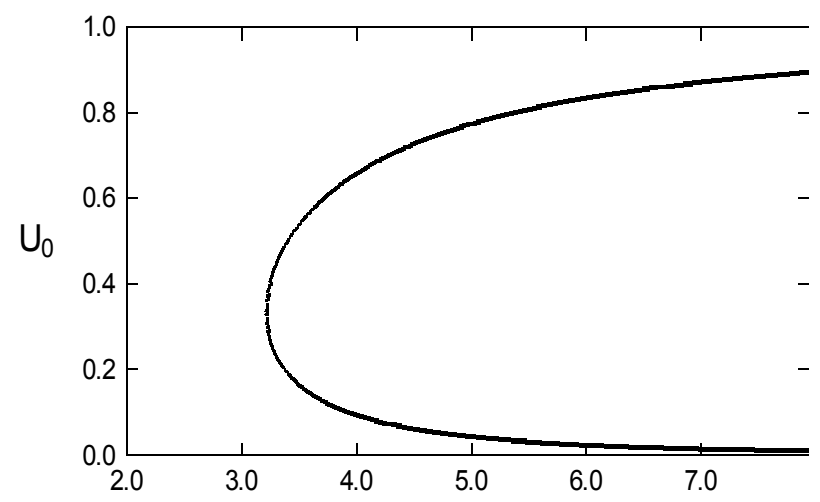

A

Figure 4. Plot of the bifurcation diagram of $U_{0}$ versus $\mathcal{A}$, as given in (2.31), characterizing the solution multiplicity of a one-spike solution for the generalized GS model (2.30). The upper branch of this curve where $1 / 3<U_{0}<1$ is unstable for all $\tau>0$, whereas solutions on the lower branch, where $0<U_{0}<1 / 3$, undergo a Hopf bifurcation at some $\tau=\tau_{H}\left(U_{0}\right)$.
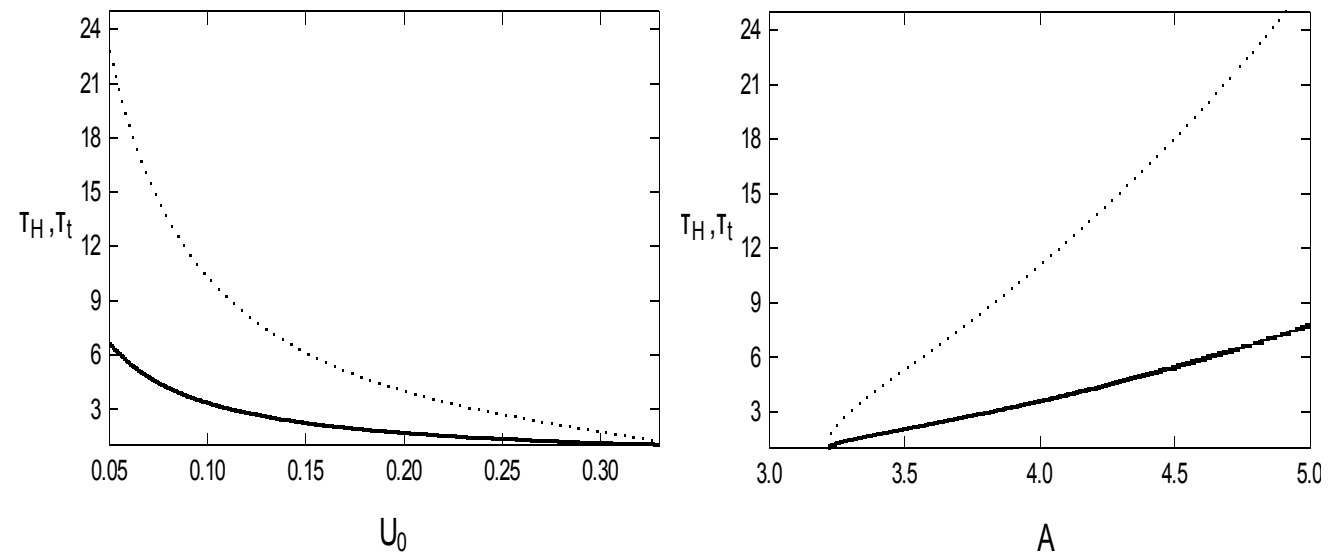

Figure 5. Plot of the thresholds $\tau_{t}$ (dotted curve) and $\tau_{H}$ (heavy solid curve), defined in (2.35), versus $U_{0}$ (left panel) and versus $\mathcal{A}$ (right panel). These correspond to the lower branch in Fig. 4. The lower curve is the Hopf bifurcation threshold, and the upper curve is where the complex conjugate pair of eigenvalues merge onto the positive real axis in the $\lambda$-plane.

grow exponentially in time with a constant growth rate. Therefore, the linearized "eigenvalue problem" no longer has its classical meaning. Instead, it should be regarded as an asymptotic theory of perturbations that evolve exponentially in time to leading order:

$$
v \sim v_{e}+e^{\lambda t} \phi(x), \quad u \sim u_{e}+e^{\lambda t} \eta ; \quad \lambda(t) \sim \lambda^{(0)}+\varepsilon^{\gamma} \lambda^{(1)}(t)+\cdots .
$$

In the discussion below $\lambda^{(0)}$ is referred to as the eigenvalue for convenience, yet one must bear in mind that only at the limit $\gamma=1$ does it in fact correspond to the classical notion. Upon substituting (2.36) into (2.3), and then collecting the leading order terms, we obtain the following expression involving the 
fractional derivative:

$$
\mathcal{S} \equiv e^{-\lambda^{(0)}} \frac{d^{\gamma}}{d t^{\gamma}} e^{\lambda^{(0)} t}=-\frac{1}{\Gamma(-\gamma)} \int_{0}^{t} \frac{1-e^{-\lambda^{(0)} \zeta}}{\zeta^{\gamma+1}} d \zeta .
$$

In the limit $t \longrightarrow \infty$ the integral converges if and only if $\operatorname{Re}\left(\lambda^{(0)}\right) \geqslant 0$, which is rather different from the behaviour with an integer derivative. With $\gamma=1$ and $\lambda^{(0)}>0$ the derivative $\frac{d^{\gamma}}{d t^{\gamma}} e^{\lambda^{(0)} t}$ diverges exponentially at $t \longrightarrow \infty$, and then the factor $\exp \left(-\lambda^{(0)} t\right)$ makes the expression finite. With $\gamma=1$ and $\lambda^{(0)}<0$ the factor $\exp \left(-\lambda^{(0)} t\right)$ diverges exponentially, but the derivative $\frac{d^{\gamma}}{d t^{\gamma}} e^{\lambda^{(0)} t}$ decays exponentially, again resulting in a finite expression. In the sub-diffusive case with $0<\gamma<1$ and $\lambda^{(0)}>0$ the derivative $\frac{d^{\gamma}}{d t^{\gamma}} e^{\lambda^{(0)} t}$ diverges exponentially at $t \longrightarrow \infty$, and the factor $\exp \left(-\lambda^{(0)} t\right)$ makes the expression finite. However for $\lambda^{(0)}<0$ the derivative $\frac{d^{\gamma}}{d t^{\gamma}} e^{\lambda^{(0)} t}$ decays only algebraically, and with the exponential factor $\exp \left(-\lambda^{(0)} t\right)$ the expression is divergent. Therefore the derivation below is valid for $\operatorname{Re}\left(\lambda^{(0)}\right) \geqslant 0$. This nuance adds a certain subtlety to the interpretation of the stability theory, rendering it sufficient to study the onset of instability, i.e. the limit $\operatorname{Re}\left(\lambda^{(0)}\right) \longrightarrow 0^{+}$, yet impossible to trace eigenvalues in the left half of the complex plane. This is summarized in the following lemma.

Lemma 2.16. In the limit $t=\sigma \varepsilon^{-(\gamma+1)} \gg 1$ with $\varepsilon \longrightarrow 0$ and $\sigma \sim \mathcal{O}(1)$ the asymptotic relation

$$
\mathcal{S} \equiv e^{-\lambda^{(0)} t} \frac{d^{\gamma}}{d t^{\gamma}} e^{\lambda^{(0)} t} \sim \lambda^{(0)^{\gamma}}+\mathcal{O}\left(\varepsilon^{\gamma(\gamma+1)}\right)
$$

holds if and only if $\operatorname{Re}\left(\lambda^{(0)}\right) \geqslant 0$.

Proof. The proof of this result was given in Lemma 3.1 of [24]. We repeat it here for the convenience of the reader. We integrate $(2.37)$ by parts and let $t=\mathcal{O}\left(\varepsilon^{-(\gamma+1)}\right) \gg 1$ to obtain

$$
\begin{gathered}
\lim _{\varepsilon \longrightarrow 0} e^{-\lambda^{(0)} t} \frac{d^{\gamma}}{d t^{\gamma}} e^{\lambda^{(0)} t}=-\frac{1}{\Gamma(1-\gamma)} \lim _{\varepsilon \longrightarrow 0}\left\{\frac{1-e^{-\lambda^{(0)} t}}{t^{\gamma}}-\lambda^{(0)} \int_{0}^{t} e^{-\lambda^{(0)} \zeta} \zeta^{-\gamma} d \zeta\right\} \sim \\
\frac{\lambda^{(0)^{\gamma}}}{\Gamma(1-\gamma)} \lim _{\varepsilon \longrightarrow 0} \int_{0}^{\lambda^{(0)} t} e^{-\xi} \xi^{-\gamma} d \xi+\mathcal{O}\left(\varepsilon^{\gamma(\gamma+1)}\right) .
\end{gathered}
$$

The error order in this expression arises from the algebraic decay of $t^{-\gamma}$ regardless of the value of $\lambda^{(0)}$. To evaluate the last integral in this expression we use contour integration. We consider the closed contour consisting of the line segment from 0 to $\lambda^{(0)} t$, an arc of radius $R \equiv\left|\lambda^{(0)}\right| t$ ( corresponding to $R \longrightarrow \infty$ at the limit $\varepsilon \longrightarrow 0)$, the interval $(\delta, R)$ on the real axis and an arc of radius $\delta \longrightarrow 0$ ( $\delta$ is independent of $\varepsilon$ ). The contour is depicted in Fig. 6. Upon using the residue theorem one gets

$$
\begin{gathered}
\lim _{\varepsilon \longrightarrow 0} e^{-\lambda^{(0)} t} \frac{d^{\gamma}}{d t^{\gamma}} e^{\lambda^{(0)} t}=\frac{\lambda^{(0)^{\gamma}}}{\Gamma(1-\gamma)} \lim _{\substack{\delta \longrightarrow 0 \\
\longrightarrow}}\left\{R^{1-\gamma} \int_{0}^{\arg \lambda^{(0)}} e^{-R e^{\imath \theta}} e^{\imath(1-\gamma) \theta} d \theta\right. \\
\left.-\imath \delta^{1-\gamma} \int_{0}^{\arg \lambda^{(0)}} e^{-\delta e^{\imath \theta}} e^{\imath(1-\gamma) \theta} d \theta+\int_{\delta}^{R} e^{-\xi} \xi^{-\gamma} d \xi\right\} \sim \lambda^{(0)^{\gamma}}+\mathcal{O}\left(\varepsilon^{\gamma(\gamma+1)}\right),
\end{gathered}
$$

where the first integral exists if and only if $\operatorname{Re}\left(\lambda^{(0)}\right) \geqslant 0$.

Remark 2.17. Since $\gamma<\gamma(\gamma+1)$ for any $0<\gamma \leqslant 1$, the asymptotic estimate above for the time scale $t=\mathcal{O}\left(\varepsilon^{-(\gamma+1)}\right)$ shows that $\mathcal{S} \sim \lambda^{(0)^{\gamma}}$ provided that $\lambda^{(0)}$ satisfies $\operatorname{Re}\left(\lambda^{(0)}\right)>0$ and is not too close to the origin in the sense that $\left|\lambda^{(0)}\right|$ must satisfy $\left|\lambda^{(0)}\right| \sim \mathcal{O}(\varepsilon)$ or larger. 


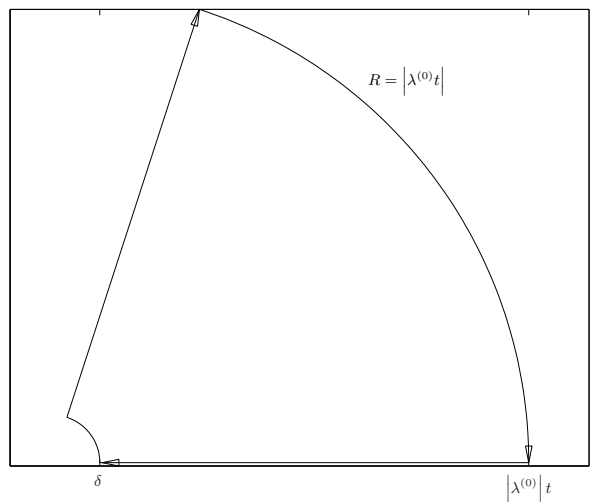

FigurE 6. Closed integration contour in the evaluation of $e^{-\lambda^{(0)}} \frac{d^{\gamma}}{d t \gamma} e^{\lambda^{(0)} t}$

From this Lemma, it follows that the spectrum of the NLEP associated with the shadow problem (2.3) is determined by the roots of (2.15a) where $\lambda$ is now replaced by $\lambda^{(0)^{\gamma}}$. By using the mapping $\lambda^{(0)^{\gamma}}=\lambda$, the previous results for the roots of (2.15a) given in Propositions 2.12-2.14 can be used to infer metastability or instability for the sub-diffusive case. More specifically, writing $\lambda=|\lambda| \exp (\imath \phi)$ with $\phi=\arg \lambda \in(-\pi, \pi]$, the map $\lambda^{(0)^{\gamma}}=\lambda$ yields that

$$
\lambda^{(0)}=|\lambda|^{1 / \gamma} e^{\imath \phi / \gamma},
$$

where the constraint $-\pi<\phi / \gamma \leqslant \pi$ must hold in order to remain on the principal branch in the $\lambda^{(0)}$ plane. Recall from Lemma 2.16, that the derivation of the NLEP for the sub-diffusive shadow problem required that $\operatorname{Re}\left(\lambda^{(0)}\right) \geqslant 0$ and $\left|\lambda^{(0)}\right| \gg \mathcal{O}(\varepsilon)$. Therefore, in terms of the spectral $\lambda$ plane associated with the regular NLEP, the sub-diffusive system will be unstable if the regular NLEP has an eigenvalue in the wedge-shaped region with cutout near the origin defined by

$$
-\frac{\pi \gamma}{2} \leqslant \phi \leqslant \frac{\pi \gamma}{2}, \quad|\lambda| \gg \mathcal{O}(\varepsilon) .
$$

This subset of the right half-plane $\operatorname{Re}(\lambda) \geqslant 0$ is shown in Fig. 7. We refer to the set (2.39) as the wedge of instability of the sub-diffusive NLEP. This wedge becomes narrower as $\gamma$ decreases, and concentrates on the positive real axis in the $\lambda$-plane as $\gamma \rightarrow 0^{+}$.

A simple consequence of this result is that if the regular NLEP admits a positive real eigenvalue $\lambda^{*}>0$, then this eigenvalue must lie in the wedge of instability for the sub-diffusive NLEP for any $0<\gamma<1$. Such an eigenvalue $\lambda^{(0)}=\left(\lambda^{*}\right)^{1 / \gamma}>0$ then yields an exponentially growing perturbation ( to leading order ) to the one-spike equilibrium solution of (2.3). Consequently, Propositions 2.12 and 2.13 also apply to the sub-diffusive case.

Proposition 2.18. Under the conditions of Propositions 2.12 and 2.13, there is an exponentially growing perturbation to the one-spike equilibrium solution of the sub-diffusive shadow problem (2.3).

Next, we consider Hopf bifurcations associated with the sub-diffusive shadow problem (2.3). The key observation is that an unstable eigenvalue of the regular NLEP only generates an instability for the sub-diffusive NLEP when it lies within the wedge of instability (2.39). This wedge of instability becomes narrower as the anomaly exponent $\gamma$ decreases. The anomaly dependent Hopf threshold, labelled by $\tau_{H \gamma}$, is computed numerically from the implicit condition that

$$
\arg \lambda=\frac{\pi \gamma}{2}
$$




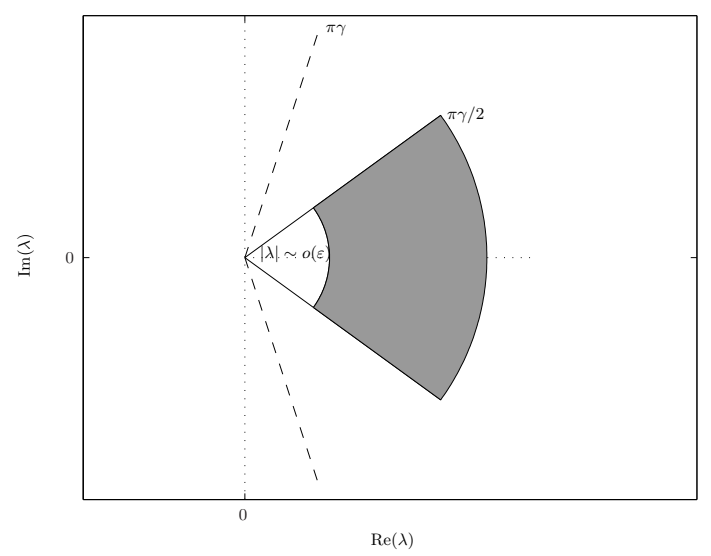

Figure 7. Admissibility and instability region in the $\lambda$ plane ( shaded ) corresponding to the asymptotics validity constraint $|\lambda| \sim \mathcal{O}(\varepsilon)$ or larger. The dashed lines show the region conforming to the principal branch.

which involves the eigenvalue path $\lambda=\lambda(\tau)$ of the roots of $(2.15 \mathrm{a})$ with $\operatorname{Re}(\lambda)>0$ and $\operatorname{Im}(\lambda)>0$. The condition (2.40) corresponds to the minimum value of $\tau$ for which this eigenvalue path enters the wedge of instability of the sub-diffusive NLEP shown in Fig. 7. In this sense, it follows that the perturbation of a one-spike solution for the sub-diffusive shadow model (2.3) is oscillatory at a larger value of $\tau$ than for the case of regular diffusion. In this sense, the effect of sub-diffusion is to stabilize the one-spike solution. This leads to the following result.

Proposition 2.19. Suppose that the assumptions $d_{0}<2, d_{1}<0$ and $\mathcal{G}(0)>2$, of Proposition 2.14 hold. Then, for all $\gamma$ in $0<\gamma<1$, the Hopf bifurcation threshold $\tau_{H \gamma}$ for the sub-diffusive NLEP must lie in the interval $\tau_{H}<\tau_{H \gamma}<\tau_{t}$. Here $\tau_{H}$ is the Hopf bifurcation threshold for the regular diffusion case, as given in (2.20), and $\tau_{t}$ is the value of $\tau$, as given in (2.18), where the complex conjugate eigenvalue pair for the regular NLEP merge onto the positive real axis. In particular, $\tau_{H \gamma} \longrightarrow \tau_{H}$ as $\gamma \longrightarrow 1^{-}$ and $\tau_{H \gamma} \longrightarrow \tau_{t}$ as $\gamma \longrightarrow 0^{+}$. In addition, if the path $\lambda=\lambda(\tau)$ of the complex eigenvalue in the first quadrant $\operatorname{Re}(\lambda)>0, \operatorname{Im}(\lambda)>0$ for the regular $N L E P$ is such that $\operatorname{Re}(\lambda)(\operatorname{Im}(\lambda))$ increases (decreases) monotonically as $\tau$ increases, then $\tau_{H \gamma}$ increases as $\gamma$ decreases.

The proof of this result follows immediately from the concept of the wedge of instability of the subdiffusive NLEP together with our explicit results in Propositions 2.12-2.14 for the the behaviour of the roots of (2.15a) in the right half-plane $\operatorname{Re}(\lambda)>0$ as a function of $\tau$.

In particular, consider the GM and generalized GS models studied in $\S 2.3$. For the generalized GS model with sub-diffusion, we conclude that the Hopf bifurcation threshold $\tau_{H \gamma}$ must lie between the two curves in Fig. 5 for any anomaly index $\gamma$. Moreover, for the sub-diffusive GM model with $s>-1$ and $q>0$, the Hopf bifurcation threshold must be in the interval $\tau_{H}<\tau_{H \gamma}<\tau_{t}$, where $\tau_{H}$ and $\tau_{t}$ are given in (2.25) and (2.26), respectively.

\section{A One-Spike Solution on the Infinite Line}

For $\varepsilon_{0} \rightarrow 0$, we use the method of matched asymptotic expansions to construct a steady-state one-spike solution to (1.2), centered at $x=0$, on the infinite line. Since the asymptotic construction is standard we will only briefly highlight the main steps of the analysis.

In the inner region near $x=0$, we introduce the new variables $y, V$, and $U$, by

$$
y=x / \varepsilon_{0}, \quad V(y)=v\left(\varepsilon_{0} y\right), \quad U(y)=u\left(\varepsilon_{0} y\right) .
$$


Upon expanding $U=U_{0}+\varepsilon_{0} U_{1}+\cdots$ and $V=V_{0}+\varepsilon_{0} V_{1}+\cdots$, and substituting into (1.2), we obtain that $U_{0}$ must be a constant and that $V_{0}$ satisfies

$$
V_{0}^{\prime \prime}-V_{0}+g_{0} V_{0}^{p}=0, \quad-\infty<y<\infty,
$$

where $g_{0} \equiv g\left(U_{0}\right)$. When $g_{0}>0$, there is a unique positive homoclinic solution for $V_{0}$ given explicitly by

$$
V_{0}(y)=\frac{1}{g_{0}^{1 /(p-1)}} w(y)
$$

where $w(y)$ is the homoclinic satisfying (2.5).

In the outer region, defined for $|x| \gg \mathcal{O}\left(\varepsilon_{0}\right)$, we obtain to all orders in $\varepsilon_{0}$ that $v=0$ and that the nonlinear term in the $u$-equation of (1.2) can be represented in terms of a Dirac mass as

$$
\varepsilon_{0}^{-1} f(u) v^{r} \rightarrow f_{0}\left(\int_{-\infty}^{\infty}\left[V_{0}(y)\right]^{r} d y\right) \delta(x)=\frac{f_{0} b_{r}}{g_{0}^{r /(p-1)}} \delta(x),
$$

where we have defined $b_{r} \equiv \int_{-\infty}^{\infty} w^{r} d y$ and $f_{0} \equiv f\left(U_{0}\right)$. In this way, we obtain that the leading-order outer solution for $u$ satisfies

$$
u_{x x}-\left(u-u_{b}\right)=-\frac{f_{0} b_{r}}{g_{0}^{r /(p-1)}} \delta(x), \quad-\infty<x<\infty ; \quad u \rightarrow u_{b} \quad \text { as } \quad|x| \rightarrow \infty .
$$

The solution for $u$ can be written as

$$
u=u_{b}+\frac{f_{0} b_{r}}{g_{0}^{r /(p-1)}} G(x)
$$

where $G(x)$ is the Green's function $G(x)$ satisfying

$$
G_{x x}-G=-\delta(x), \quad-\infty<x<\infty ; \quad G \rightarrow 0 \quad \text { as } \quad|x| \rightarrow \infty,
$$

which is given explicitly by $G(x)=e^{-|x|} / 2$.

Then, to match inner and outer solutions for $u$ we must set $U_{0}=u(0)$, which yields a nonlinear algebraic equation for $U_{0}$ given by

$$
U_{0}-u_{b}=\frac{f_{0} b_{r}}{2 g_{0}^{r /(p-1)}}, \quad f_{0} \equiv f\left(U_{0}\right), \quad g_{0} \equiv g\left(U_{0}\right), \quad b_{r} \equiv \int_{-\infty}^{\infty} w^{r} d y .
$$

We summarize the construction of the steady-state one-spike solution for (1.2) as follows:

Proposition 3.1. For $\varepsilon_{0} \rightarrow 0$, a one-spike equilibrium solution to (1.2), which we label by $v_{e}(x)$ and $u_{e}(x)$, is given asymptotically by

$$
v_{e}(x) \sim \frac{1}{g_{0}^{1 /(p-1)}} w\left(x / \varepsilon_{0}\right), \quad u_{e}(x) \sim u_{b}+\left(U_{0}-u_{b}\right) \frac{G(x)}{G(0)},
$$

where $U_{0}$ is a solution to the nonlinear algebraic equation (3.4) and where $G(x)=\frac{1}{2} e^{-|x|}$ is the Green's function satisfying (3.3). This construction is valid provided that $g_{0} \equiv g\left(U_{0}\right)>0$.

To analyze the linearized stability of this one-spike solution we set

$$
v=v_{e}+e^{\lambda t} \phi, \quad u=u_{e}+e^{\lambda t} \eta .
$$


Upon substituting (3.6) into (1.2) and linearizing, we obtain the eigenvalue problem

$$
\begin{gathered}
\varepsilon_{0}^{2} \phi_{x x}-\phi+p g\left(u_{e}\right) v_{e}^{p-1} \phi+g^{\prime}\left(u_{e}\right) v_{e}^{p} \eta=\lambda \phi, \\
\eta_{x x}-(1+\tau \lambda) \eta=-\varepsilon_{0}^{-1}\left[f^{\prime}\left(u_{e}\right) v_{e}^{r} \eta+r f\left(u_{e}\right) v_{e}^{r-1} \phi\right] .
\end{gathered}
$$

Since $v_{e}$ is localized near $x=0$, we look for a localized eigenfunction for $\phi(x)$ in the form $\phi=\Phi(y)$ where $y=x / \varepsilon_{0}$. Upon using $v_{e} \sim g_{0}^{-1 /(p-1)} w$ and $u_{e} \sim U_{0}$ for $x=\mathcal{O}\left(\varepsilon_{0}\right)$, we obtain from (3.7a) that $\Phi(y)$ satisfies

$$
L_{0} \Phi+\frac{g_{0}^{\prime}}{g_{0}^{p /(p-1)}} w^{p} \eta(0)=\lambda \Phi, \quad-\infty<y<\infty ; \quad \Phi \rightarrow 0 \quad \text { as } \quad|y| \rightarrow \infty .
$$

Here we have labelled $g_{0}^{\prime} \equiv g^{\prime}\left(U_{0}\right)$. In addition, $L_{0}$ is the local operator defined by $(2.8)$.

To derive our NLEP we must calculate $\eta(0)$ in (3.8). Since $\phi$ is localized near $x=0$, then for $\varepsilon_{0} \rightarrow 0$ the right-hand side of $(3.7 \mathrm{~b})$ can be calculated in the sense of distributions by using $u_{e} \sim U_{0}$ and $v_{e} \sim g_{0}^{-1 /(p-1)} w$ as

$$
\varepsilon_{0}^{-1}\left[f^{\prime}\left(u_{e}\right) v_{e}^{r} \eta+f\left(u_{e}\right) r v_{e}^{r-1} \phi\right] \rightarrow\left[\frac{\eta(0) f_{0}^{\prime}}{g_{0}^{r /(p-1)}} \int_{-\infty}^{\infty} w^{r} d y+\frac{r f_{0}}{g_{0}^{(r-1) /(p-1)}} \int_{-\infty}^{\infty} w^{r-1} \Phi d y\right] \delta(x),
$$

where we have defined $f_{0}^{\prime} \equiv f^{\prime}\left(U_{0}\right)$. In this way, we obtain that the outer approximation for $\eta$ satisfies

$$
\begin{aligned}
\eta_{x x}-(1+\tau \lambda) \eta & =-\frac{\eta(0) f_{0}^{\prime} b_{r}}{g_{0}^{r /(p-1)}} \delta(x)-\frac{r f_{0}}{g_{0}^{(r-1) /(p-1)}}\left(\int_{-\infty}^{\infty} w^{r-1} \Phi d y\right) \delta(x), \quad-\infty<x<\infty \\
\eta \rightarrow 0 \quad \text { as } \quad|x| \rightarrow \infty &
\end{aligned}
$$

We represent the solution to (3.9) in terms of the $\lambda$-dependent Green's function $G_{\lambda}(x)$ satisfying

$$
G_{\lambda x x}-(1+\tau \lambda) G_{\lambda}=-\delta(x), \quad-\infty<x<\infty ; \quad G_{\lambda} \rightarrow 0 \quad \text { as } \quad|x| \rightarrow \infty,
$$

which has the explicit solution

$$
G_{\lambda}(x)=\frac{1}{2 \sqrt{1+\tau \lambda}} e^{-|x| \sqrt{1+\tau \lambda}},
$$

provided that we take the principal branch of the square root function to guarantee that $\operatorname{Re}(\sqrt{1+\tau \lambda})>0$ and, hence, ensure decay of $G_{\lambda}(x)$ as $|x| \rightarrow \infty$ for any complex $\lambda$.

In terms of $G_{\lambda}(x)$, the solution to (3.9) is

$$
\eta(x)=\frac{\eta(0) f_{0}^{\prime} b_{r}}{g_{0}^{r /(p-1)}} G_{\lambda}(x)+\frac{r f_{0}}{g_{0}^{(r-1) /(p-1)}}\left(\int_{-\infty}^{\infty} w^{r-1} \Phi d y\right) G_{\lambda}(x) .
$$

We then set $x=0$ in (3.11) and solve for $\eta(0)$. Finally, upon substituting $\eta(0)$ into (3.8) and re-writing the resulting expression by using (3.4), we obtain an NLEP for $\Phi(y)$. The result is summarized as follows:

Proposition 3.2. For $0<\varepsilon_{0} \ll 1$, the stability of the one-spike steady-state solution of Proposition 3.1 is determined by the spectrum of the NLEP (2.9a), where the multiplier of the nonlocal term is given by

$$
\chi=\frac{r g_{0}^{\prime}}{g_{0}}\left[\frac{f_{0}^{\prime}}{f_{0}}-\frac{1}{U_{0}-u_{b}} \frac{G(0)}{G_{\lambda}(0)}\right]^{-1} .
$$

In this expression, the ratio of the two Green's functions at $x=0$ is simply

$$
\frac{G(0)}{G_{\lambda}(0)}=\sqrt{1+\tau \lambda}
$$




\subsection{NLEP Stability Theory with Regular Diffusion}

In this section we obtain explicit stability results for the NLEP of Proposition 3.2 for the explicitly solvable case where $p=2 r-3$ and $r>2$. For this case, we need only analyze the roots of (2.13) where $\chi(\lambda)$ is defined in (3.12a). By using (3.12a) for $\chi$ and (3.4) for $U_{0}$, we can write (2.13) more conveniently in the following form:

Proposition 3.3. For $p=2 r-3$ and $r>2$, the discrete eigenvalues $\lambda$ of the NLEP in Proposition 3.2 are the roots of the transcendental equation

$$
2 \sqrt{1+\tau \lambda}=\mathcal{G}(\lambda) \equiv d_{0}-\frac{d_{1}}{\beta-\lambda},
$$

where $\beta=r^{2}-2 r>0$. Here $d_{0}$ and $d_{1}$ are defined in (2.15b). In (3.13), $\sqrt{1+\tau \lambda}$ denotes the principal branch of the square root. In terms of $d_{0}$ and $d_{1}$, the multiplier $\chi$ of the NLEP in Proposition 3.2 is

$$
\chi=\frac{2}{r}\left(\frac{d_{1}}{d_{0}-2 \sqrt{1+\tau \lambda}}\right)
$$

When $d_{0}>2$, this multiplier has a simple pole at $\lambda=\tau^{-1}\left(-1+d_{0}^{2} / 4\right)$, which lies in $\operatorname{Re}(\lambda)>0$.

Remark 3.4. The transcendental equation (3.13) has precisely the same form as for the one-spike case for the shadow problem given in (2.15a), provided that we simply replace $\sqrt{1+\tau \lambda}$ in $(3.13)$ with $(1+\tau \lambda)$.

We first determine sufficient conditions for instability by characterizing the roots of (3.13) on the positive real axis. Since the proofs of these results parallel that of Propositions 2.12 and 2.13 for the shadow problem (2.2), they are omitted.

Proposition 3.5. Suppose that either

$$
(I) \quad d_{1}<0 \text { and } \mathcal{G}(0)<2 ; \quad \text { or } \quad(I I) \quad d_{1}>0 \text { and } \mathcal{G}(0)>2 ; \quad \text { or } \quad(I I I) \quad d_{1}>0 \text { and } d_{0}<2 .
$$

Then, for any $\tau>0$, (3.13) has at least one root with $\lambda>0$ and $\lambda$ real. Thus, the NLEP of Proposition 3.2 has at least one unstable real eigenvalue and the equilibrium spike solution for (1.2) is unstable.

Proposition 3.6. Suppose that $d_{1}<0, \mathcal{G}(0)>2$ and $d_{0}<2$. Then, when $\tau>0$ is sufficiently large, (3.13) has two real positive roots in $0<\lambda<\beta$, while for $\tau>0$ sufficiently small (3.13) has no positive real roots. Next, if $d_{1}<0$ and $d_{0}>2$, so that necessarily $\mathcal{G}(0)>2$, then (3.13) has two real roots in $\lambda>\beta$ when $\tau>0$ is sufficiently small, and two real roots in $0<\lambda<\beta$ when $\tau>0$ is sufficiently large.

Next, we count the number $N$ of roots of (3.13) in $\operatorname{Re}(\lambda)>0$. We define $\mathcal{F}(\lambda) \equiv 2 \sqrt{1+\tau \lambda}-\mathcal{G}(\lambda)$, where $\mathcal{G}(\lambda)$ has a simple pole in $\operatorname{Re}(\lambda)>0$ at $\lambda=\beta$. We take the counterclockwise contour consisting of the imaginary axis $-i R \leq \operatorname{Im} \lambda \leq i R$ and the semi-circle $\Gamma_{R}$, given by $|\lambda|=R>0$, for $-\pi / 2 \leq \arg \lambda \leq \pi / 2$. For $\tau>0$, we have that $\mathcal{F}(\lambda) \sim 2 \sqrt{\tau \lambda}$ as $|\lambda| \rightarrow \infty$ on $\Gamma_{R}$, so that the change in the argument of $\mathcal{F}$ over $\Gamma_{R}$ as $R \rightarrow \infty$ is $\pi / 2$. By using the argument principle, together with $\mathcal{F}(\bar{\lambda})=\overline{\mathcal{F}(\lambda)}$, we obtain for $\tau>0$ that

$$
N=\frac{5}{4}+\frac{1}{\pi}[\arg \mathcal{F}]_{\Gamma_{I}},
$$

where $[\arg \mathcal{F}]_{\Gamma_{I}}$ denotes the change in the argument of $\mathcal{F}$ along the semi-infinite imaginary axis $\Gamma_{I}=i \lambda_{I}$, $0 \leq \lambda_{I}<\infty$, traversed in the downwards direction. Now along the imaginary axis $\lambda=i \lambda_{I}$ for $\lambda_{I}>0$, we can readily separate $\mathcal{F}\left(i \lambda_{I}\right)$ in terms of real and imaginary parts as $\mathcal{F}\left(i \lambda_{I}\right)=\mathcal{F}_{R}\left(\lambda_{I}\right)+i \mathcal{F}_{I}\left(\lambda_{I}\right)$, to obtain that

$$
\mathcal{F}_{R}\left(\lambda_{I}\right)=\mathcal{K}_{+}\left(\tau \lambda_{I}\right)-d_{0}+\frac{d_{1} \beta}{\beta^{2}+\lambda_{I}^{2}}, \quad \mathcal{F}_{I}\left(\lambda_{I}\right)=\mathcal{K}_{-}\left(\tau \lambda_{I}\right)+\frac{d_{1} \lambda_{I}}{\beta^{2}+\lambda_{I}^{2}},
$$


where we have defined $\mathcal{K}_{ \pm}(\zeta)$ by

$$
\mathcal{K}_{ \pm}(\zeta)=\sqrt{2}\left[\sqrt{1+\zeta^{2}} \pm 1\right]^{1 / 2}
$$

For any $\tau>0$, we have that $\mathcal{F}_{R} \sim \sqrt{2 \tau \lambda_{I}}$ and $\mathcal{F}_{I} \sim \sqrt{2 \tau \lambda_{I}}$ as $\lambda_{I} \rightarrow+\infty$, so that $\arg \left(\mathcal{F}\left(i \lambda_{I}\right)\right) \rightarrow \pi / 4$ as $\lambda_{I} \rightarrow+\infty$. Alternatively, for $\lambda_{I}=0$, we obtain

$$
\mathcal{F}_{R}(0)=2-\mathcal{G}(0), \quad \mathcal{F}_{I}(0)=0 .
$$

For $\tau>0$, we calculate that

$$
\frac{d}{d \lambda_{I}} \mathcal{F}_{R}\left(\lambda_{i}\right)=\tau \mathcal{K}_{+}^{\prime}\left(\tau \lambda_{I}\right)-\frac{2 d_{1} \lambda_{I} \beta}{\left(\beta^{2}+\lambda_{I}\right)^{2}}
$$

By using these preliminary results, we can calculate $[\arg \mathcal{F}]_{\Gamma_{I}}$, and thus obtain $N$ from (3.16). Our first result is as follows:

Proposition 3.7. Let $d_{1}<0$ and $\mathcal{G}(0)>2$, and let $N$ denote the number of roots to (3.13) in $\operatorname{Re}(\lambda)>0$. Then, for all $\tau>0$, we have either $N=0$ or $N=2$. Moreover, if $d_{0}<2$, then $N=2$ for $\tau \gg 1$ and $N=0$ for $\tau \ll 1$.

Proof. Since $d_{1}<0$ and $\mathcal{K}_{+}^{\prime}(\zeta)>0$, then from (3.19) we conclude that $\frac{d}{d \lambda_{I}} \mathcal{F}_{R}\left(\lambda_{I}\right)>0$. Next, since $\mathcal{G}(0)>2$, we have from $(3.18)$ that $\mathcal{F}_{R}(0)<0$, while $\mathcal{F}_{R}(+\infty)=+\infty$. Therefore, there exists a unique root $\lambda_{I}^{*}>0$, with $\lambda_{I}^{*}=\lambda_{I}^{*}(\tau)$, for which $\mathcal{F}_{R}\left(\lambda_{I}^{*}\right)=0$. It then follows that $[\arg \mathcal{F}]_{\Gamma_{I}}=3 \pi / 4$ if $\mathcal{F}_{I}\left(\lambda_{I}^{*}\right)>0$ and $[\arg \mathcal{F}]_{\Gamma_{I}}=-5 \pi / 4$ if $\mathcal{F}_{I}\left(\lambda_{I}^{*}\right)>0$. We conclude from (3.16) that either $N=2$ or $N=0$ depending on whether $\mathcal{F}_{I}\left(\lambda_{I}^{*}\right)$ is positive or negative, respectively. To determine whether $N=0$ or $N=2$, we must calculate the root $\lambda_{I}^{*}$ to $\mathcal{F}_{R}\left(\lambda_{I}\right)=0$. From using $(3.17 \mathrm{a})$ for $\mathcal{F}_{R}\left(\lambda_{I}\right)$, we obtain that $\lambda_{I}^{*}$ is the unique root of

$$
\mathcal{K}_{+}\left(\tau \lambda_{I}\right)=\mathcal{R}\left(\lambda_{I}\right) \equiv d_{0}-\frac{d_{1} \beta}{\beta^{2}+\lambda_{I}^{2}} .
$$

Suppose that $d_{0}<2, d_{1}<0$, and $\mathcal{G}(0)>2$. Then, for $\tau \ll 1$, (3.20) has a root with $\lambda_{I}^{*} \sim \lambda_{I 0}=\mathcal{O}(1)$, where $\lambda_{I 0}$ satisfies

$$
2-d_{0}+\frac{d_{1} \beta}{\beta^{2}+\lambda_{I 0}^{2}}=0
$$

so that

$$
\lambda_{I}^{*}(0)=\lambda_{I 0} \equiv \beta \sqrt{\frac{(\mathcal{G}(0)-2)}{2-d_{0}}} .
$$

For $\tau \ll 1$, we conclude from (3.17a) that $\mathcal{F}_{I}\left(\lambda_{I 0}\right)=d_{1} \lambda_{I 0} /\left(\beta^{2}+\lambda_{I 0}^{2}\right)+\mathcal{O}\left(\tau^{2}\right)<0$. Therefore, $N=0$ when $\tau \ll 1$. Alternatively, for $\tau \gg 1$, the unique root of (3.20) satisfies $\lambda_{I}^{*} \sim \lambda_{I \infty} / \tau$, where $\lambda_{I \infty}$ is the unique root of $\mathcal{K}_{+}\left(\lambda_{I \infty}\right)=\mathcal{G}(0)>2$. From (3.17a), we conclude that $\mathcal{F}_{I}\left(\lambda_{I}^{*}\right) \sim \mathcal{K}_{-}\left(\lambda_{I \infty}\right)+\mathcal{O}\left(\tau^{-1}\right)>0$. We conclude that $N=2$ when $\tau \gg 1$.

Remark 3.8. For the case where $d_{1}<0, \mathcal{G}(0)>2$ and $d_{0}<2$, Proposition 3.7 proves the existence of a value of $\tau$ for which a one-spike solution of (1.2) undergoes a Hopf bifurcation. Under these conditions on $d_{0}$ and $d_{1}, N=0$ for $\tau \ll 1$, and $N=2$ for $\tau \gg 1$. respectively. Therefore, by continuity of the path of eigenvalues as a function of $\tau$, there must be a value $\tau_{H}$ of $\tau$ for which (3.13) has a complex conjugate pair of roots $\lambda= \pm i \lambda_{I}$ with $\lambda_{I}>0$.

The next result gives a sufficient condition for the existence of a unique value $\tau_{H}>0$ of $\tau$ for which (3.13) has a complex conjugate pair of roots. These roots cross transversally into $\operatorname{Re}(\lambda)>0$ when $\tau>\tau_{H}$. This leads to a rigorous Hopf bifurcation result for one-spike solutions of (1.2). 
Proposition 3.9. Suppose that $d_{1}<0, d_{0}<2$ and $2<\mathcal{G}(0)<4-d_{0}$ where $\mathcal{G}(0)=d_{0}-d_{1} / \beta$. Then, there is a unique value $\tau=\tau_{H}>0$ at which the NLEP of Proposition 3.2 undergoes a Hopf bifurcation. The one-spike solution is spectrally stable if $0<\tau<\tau_{H}$ and is spectrally unstable if $\tau>\tau_{H}$.

Proof. We write $\mathcal{F}_{R}$ in (3.17a) as $\mathcal{F}_{R}\left(\lambda_{I} ; \tau\right)$. Since $\mathcal{G}(0)>2$ and $d_{1}<0$, then as shown in the proof of Proposition 3.7, there is a unique root $\lambda_{I}^{*}(\tau)$ to $\mathcal{F}_{R}=0$, so that $\mathcal{F}_{R}\left(\lambda_{I}^{*}(\tau) ; \tau\right)=0$. At this root, we define $\mathcal{N}(\tau) \equiv \mathcal{F}_{I}\left(\lambda_{I}^{*}(\tau) ; \tau\right)$. In the proof of Proposition 3.7 we showed that $\mathcal{N}(\tau)<0$ for $0<\tau \ll 1$ when $d_{0}<2$, and $\mathcal{N}(\tau) \rightarrow+\infty$ as $\tau \rightarrow+\infty$. We will now show that $\mathcal{N}^{\prime}(\tau)>0$ for all $\tau>0$ when $d_{0}<2$ and $2<\mathcal{G}(0)<4-d_{0}$. Such a monotonicity result would establish the existence of a unique Hopf bifurcation value $\tau_{H}>0$ at which $\mathcal{N}\left(\tau_{H}\right)=0$, with $\mathcal{N}(\tau)>0$ for $\tau>\tau_{H}$ and $\mathcal{N}(\tau)<0$ for $0<\tau<\tau_{H}$. Therefore, $N=2$ when $\tau>\tau_{H}$ and $N=0$ when $0<\tau<\tau_{H}$. Thus, to complete the proof we need only find sufficient conditions that guarantee that $\mathcal{N}^{\prime}(\tau)>0$ for all $\tau$.

To determine the sign of $\mathcal{N}^{\prime}(\tau)$, we set $\frac{d}{d \tau} \mathcal{F}_{R}\left(\lambda_{I}^{*}(\tau) ; \tau\right)=0$, and then use $(3.17 \mathrm{a})$ for $\mathcal{F}_{R}$ to get

$$
\lambda_{I}^{*}=-\frac{d \mathcal{F}_{R} / d \tau}{d \mathcal{F}_{R} / d \lambda_{I}}=\frac{-\lambda_{I}^{*} \mathcal{K}_{+}^{\prime}}{\tau \mathcal{K}_{+}^{\prime}-2 d_{1} \beta \lambda_{I}^{*}\left(\beta^{2}+\left(\lambda_{I}^{*}\right)^{2}\right)^{-2}} .
$$

Then, we use $(3.17 \mathrm{a})$ for $\mathcal{F}_{I}$ to calculate $\mathcal{N}^{\prime}(\tau)=\lambda_{I}^{* \prime} d \mathcal{F}_{I} / d \lambda_{I}+d \mathcal{F}_{I} / d \tau$. After a short calculation, we obtain

$$
\mathcal{N}^{\prime}(\tau)=-\left.\frac{d_{1}\left(\beta^{2}+\lambda_{I}^{2}\right)\left[\mathcal{K}_{+}^{\prime} \lambda_{I}\left(\beta^{2}-\lambda_{I}^{2}\right)+2 \beta \lambda_{I}^{2} \mathcal{K}_{-}^{\prime}\right]}{\tau \mathcal{K}_{+}^{\prime}\left(\beta^{2}+\lambda_{I}\right)^{2}-2 d_{1} \beta \lambda_{I}}\right|_{\lambda_{I}=\lambda_{I}^{*}} .
$$

Since $d_{1}<0$ and $\mathcal{K}_{ \pm}^{\prime}>0$, a sufficient condition to ensure that $\mathcal{N}^{\prime}(\tau)>0$ for all $\tau>0$ is that $\lambda_{I}^{*}(\tau)<\beta$ for all $\tau>0$. Since $\lambda_{I}^{* \prime}(\tau)<0$, this condition holds for all $\tau>0$ when $\lambda_{I}^{*}(0)<\beta$. Finally, we recall from (3.21) that when $d_{1}<0$ and $d_{0}<2$ we have $\left(\lambda_{I}^{*}(0)\right)^{2}+\beta^{2}=d_{1} \beta /\left(d_{0}-2\right)>0$. Therefore, $\lambda_{I}^{*}(0)<\beta$ when $d_{1} \beta /\left(d_{0}-2\right) \leq 2 \beta^{2}$. This implies that $\mathcal{G}(0)=d_{0}-d_{1} / \beta<4-d_{0}$. This completes the proof of the result.

Remark 3.10. The previous NLEP stability analyses for the GM and GS models have not been successful in providing a rigorous proof for the existence of a unique Hopf bifurcation value of $\tau$ (see $\S 6$ of [37]). Only for the special case of the shadow GM system is such a result available for certain exponent sets of the GM nonlinearities (cf. [38]). Our Proposition 3.9 provides sufficient conditions on the nonlinearities $f(u)$ and $g(u)$ for the uniqueness of the Hopf bifurcation point for a one-spike solution of the general system (1.2) for the case $p=2 r-3$ and $r>2$ for which the associated NLEP is explicitly solvable.

We remark that the proof of Proposition 3.9 does not require detailed knowledge of the functions $\mathcal{K}_{ \pm}(\zeta)$ with $\zeta=\tau \lambda_{I}$. It only requires that $\mathcal{K}_{ \pm}^{\prime}>0$ and that $\mathcal{K}_{+}(0)=2$.

Proposition 3.9 provides sufficient conditions on $d_{0}$ and $d_{1}$ for the existence of a unique Hopf bifurcation point. However, by using detailed properties of the functions $\mathcal{K}_{ \pm}$, we now show that the conditions in Proposition 3.9 are in fact not necessary, and that a unique Hopf bifurcation point occurs for any $\mathcal{G}(0)$ with $\mathcal{G}(0)>2$. Our result is as follows:

Proposition 3.11. Suppose that $d_{1}<0, d_{0}<2$ and $\mathcal{G}(0)>2$. Then, the NLEP of Proposition 3.2 undergoes a Hopf bifurcation at $\tau=\tau_{H}>0$, where

$$
\tau_{H}=\frac{d_{1}^{2}}{2 \xi^{2}} A, \quad \text { where } \quad A \equiv \beta-\frac{d_{0} \xi}{d_{1}},
$$

and where $\xi$ is the smallest root of the quadratic equation

$$
\mathcal{M}(\xi) \equiv\left(d_{0}^{2}-4\right) \xi^{2}-\left(d_{1}^{2}+2 \beta d_{0} d_{1}\right) \xi+2 \beta^{2} d_{1}^{2}=0,
$$

on the interval $\xi>\beta^{2}$. In terms of this root, the corresponding eigenvalue is $\lambda=i \lambda_{I H}$, where

$$
\lambda_{I H}=\sqrt{\xi-\beta^{2}} .
$$


For the special case $d_{0}=0, \tau_{H}$ is given explicitly by

$$
\tau_{H}=\frac{1}{\beta}\left[1+\frac{c^{2}}{2}+c \sqrt{1+\frac{c^{2}}{4}}\right], \quad c \equiv-\frac{d_{1}}{2 \beta \sqrt{2}}>\frac{1}{\sqrt{2}},
$$

while the corresponding eigenvalue is $\lambda=i \lambda_{I H}$, where

$$
\lambda_{I H}=\beta \sqrt{1-\frac{2}{\beta \tau_{H}}} .
$$

Proof. In (3.17a), we set $\mathcal{F}_{R}=\mathcal{F}_{I}=0$ to obtain

$$
\sqrt{2}[\sqrt{a}+1]^{1 / 2}=d_{0}-\frac{d_{1} \beta}{\xi}, \quad \sqrt{2}[\sqrt{a}-1]^{1 / 2}=-\frac{d_{1} \lambda_{I}}{\xi} ; \quad a \equiv 1+\tau^{2} \lambda_{I}^{2}, \quad \xi \equiv \beta^{2}+\lambda_{I}^{2} .
$$

Upon dividing the resulting two expressions we get

$$
\left(\frac{\sqrt{a}+1}{\sqrt{a}-1}\right)^{1 / 2}=\frac{1+\sqrt{a}}{\lambda_{I} \tau}=\frac{\beta}{\lambda_{I}}-\frac{d_{0} \xi}{d_{1} \lambda_{I}},
$$

which reduces to

$$
\sqrt{a}+1=\tau A, \quad \text { where } \quad A \equiv \beta-\frac{d_{0} \xi}{d_{1}} .
$$

Since the first equation of $(3.25)$ is $\sqrt{2}[\sqrt{a}+1]^{1 / 2}=-d_{1} A / \xi$, we obtain from using (3.26) that $\sqrt{2 \tau} A^{1 / 2}=$ $-d_{1} A / \xi$. Upon solving for $\tau$, and recalling that $A \equiv \beta-d_{0} \xi / d_{1}$, we obtain

$$
\tau=\frac{d_{1}^{2}}{2 \xi^{2}} A=\frac{d_{1}^{2}}{2}\left(\frac{\beta}{\xi^{2}}-\frac{d_{0}}{\xi d_{1}}\right),
$$

which is (3.23a). Equation (3.27) determines $\tau$ in terms of $\xi$. To obtain the quadratic equation (3.23b) for $\xi$, we square and add the two expressions in (3.25) to obtain

$$
\sqrt{a}=\frac{1}{4}\left[\left(d_{0}-\frac{d_{1} \beta}{\xi}\right)^{2}+\frac{d_{1}^{2} \lambda_{I}^{2}}{\xi^{2}}\right] .
$$

Then, by using $\sqrt{a}=-1+\tau A, \lambda_{I}^{2}=\xi-\beta^{2}$, and (3.27) for $\tau$ in terms of $A$, (3.28) becomes

$$
\frac{A^{2} d_{1}^{2}}{2 \xi^{2}}-1=\frac{1}{4}\left[\frac{d_{1}^{2} A^{2}}{\xi^{2}}+\frac{d_{1}^{2}}{\xi^{2}}\left(\xi-\beta^{2}\right)\right] .
$$

Upon solving for $A^{2}$ and recalling that $A=\beta-d_{0} \xi / d_{1}$, we obtain

$$
\frac{4 \xi^{2}}{d_{1}^{2}}+\xi-\beta^{2}=A^{2}=\left(\beta-d_{0} \xi / d_{1}\right)^{2} .
$$

By rewriting this last expression we conclude that $\xi>\beta^{2}$ must be a root of the quadratic equation of (3.23b).

We must now carefully examine the roots of $\mathcal{M}(\xi)=0$ when $d_{0}<2, d_{1}<0$, and $\mathcal{G}(0)=d_{0}-d_{1} / \beta>2$. There are three sub-cases that must be considered: Case 1: $\left|d_{0}\right|<2$; Case 2: $d_{0}=-2$; Case $3: d_{0}<-2$. For each of these cases we calculate that

$$
\begin{aligned}
\mathcal{M}\left(\beta^{2}\right) & =\left(d_{0}^{2}-4\right) \beta^{4}-\left(d_{1}^{2}+2 \beta d_{0} d_{1}\right) \beta^{2}+2 \beta^{2} d_{1}^{2} \\
& =-4 \beta^{4}+\beta^{4}\left(\frac{d_{1}^{2}}{\beta^{2}}-\frac{2 d_{0} d_{1}}{\beta}+d_{0}^{2}\right)=\beta^{4}\left([\mathcal{G}(0)]^{2}-4\right) .
\end{aligned}
$$


Since $\mathcal{G}(0)>2$, we conclude that $\mathcal{M}\left(\beta^{2}\right)>0$.

For Case I where $\left|d_{0}\right|<2$, we have that $\mathcal{M}(\xi) \rightarrow-\infty$ as $\xi \rightarrow \pm \infty$. Therefore, by the intermediate value theorem there exists a unique root $\xi_{-}$to $\mathcal{M}(\xi)=0$ in $\beta^{2}<\xi<\infty$, while the other root is in $-\infty<\xi<\beta^{2}$. Since $\xi=\beta^{2}+\lambda_{I}^{2}$, the relevant root is $\xi_{-}$. Next, we must show that $A=\beta-d_{0} \xi_{-} / d_{1}>0$, so that $\tau_{H}>0$ from (3.23a). If $0<d_{0}<2$, then $A>0$ since $d_{0}>0, \xi_{-}>0$ and $d_{1}<0$. For $-2<d_{0}<0$, we use $\mathcal{G}(0)>2$ to obtain $d_{1} / \beta<d_{0}-2$. Therefore, since $d_{0}<0$ and $\xi_{-}>0$, we estimate $-d_{0} \xi_{-} / d_{1}>-d_{0} \xi_{-} /\left(\beta\left(d_{0}-2\right)\right.$. By using this inequality we obtain

$$
A=\beta-\frac{d_{0} \xi_{-}}{d_{1}}>\beta-\frac{d_{0} \xi_{-}}{\beta\left(d_{0}-2\right)}=\frac{d_{0}\left(\xi_{-}-\beta^{2}\right)+2 \beta^{2}}{\beta\left(2-d_{0}\right)}>0,
$$

since $-2<d_{0}<0$ and $\xi_{-}>\beta^{2}$. Therefore, when $\left|d_{0}\right|<2,(3.23 \mathrm{~b})$ has a unique root in $\xi>\beta^{2}$, for which $\tau_{H}>0$ in $(3.23 \mathrm{a})$.

For the degenerate Case II where $d_{0}=-2$, we have $\mathcal{M}(\xi)=-\left(d_{1}^{2}-4 \beta d_{1}\right) \xi+2 \beta^{2} d_{1}^{2}$. Since $d_{1}<0$, then $\mathcal{M}(\xi) \rightarrow-\infty$ as $\xi \rightarrow+\infty$, while $\mathcal{M}\left(\beta^{2}\right)>0$ from $(3.29 \mathrm{~b})$. Therefore, the unique root $\xi=$ $2 \beta^{2} d_{1} /\left(d_{1}-4 \beta\right)$ to $\mathcal{M}(\xi)=0$ is in $\xi>\beta^{2}$. At this root we calculate $A$ as

$$
A=\beta-\frac{d_{0} \xi}{d_{1}}=\beta+\frac{2 \xi}{d_{1}}=\beta+\frac{4 \beta^{2}}{d_{1}-4 \beta}=\frac{\beta d_{1}}{d_{1}-4 \beta}>0
$$

since $d_{1}<0$. Thus, $\tau_{H}>0$ in $(3.23 \mathrm{a})$.

Finally, we consider Case III where $d_{0}<-2$. This case is more delicate since $\mathcal{M}(\xi) \rightarrow+\infty$ as $\xi \rightarrow+\infty$ and $\mathcal{M}\left(\beta^{2}\right)>0$. Therefore, the behaviour of the roots in $\xi>\beta^{2}$ is not immediately clear. However, we now show that $\mathcal{M}\left(\xi_{c}\right)<0$ where $\xi_{c} \equiv d_{1} \beta / d_{0}$ is the unique root of $A=0$. For $\xi<\xi_{c}$ we have $A>0$, while for $\xi>\xi_{c}$, we have $A<0$. We readily find that $\xi_{c}>\beta^{2}$ since $d_{0}-d_{1} / \beta>2$. To establish the sign of $\mathcal{M}\left(\xi_{c}\right)$ we calculate

$$
\begin{aligned}
\mathcal{M}\left(\xi_{c}\right) & =\left(d_{0}^{2}-4\right) \frac{d_{1}^{2} \beta^{2}}{d_{0}^{2}}-\left(d_{1}^{2}+2 d_{0} d_{1} \beta\right) \frac{d_{1} \beta}{d_{0}}+2 \beta^{2} d_{1}^{2} \\
& =d_{1}^{2} \beta^{2}-\frac{4 d_{1}^{2} \beta^{2}}{d_{0}^{2}}-\frac{d_{1}^{3} \beta}{d_{0}}=\frac{d_{1}^{2} \beta^{2}}{d_{0}^{2}}\left[d_{0}^{2}-4-\frac{d_{1} d_{0}}{\beta}\right] .
\end{aligned}
$$

Since $d_{0}-d_{1} / \beta>2$ and $d_{0}<0$, we have $-d_{0} d_{1} / \beta<d_{0}\left(2-d_{0}\right)$. By using this estimate in the last expression above, we obtain

$$
\mathcal{M}\left(\xi_{c}\right)<\frac{d_{1}^{2} \beta^{2}}{d_{0}^{2}}\left[d_{0}^{2}-4+2 d_{0}-d_{0}^{2}\right]=\frac{d_{1}^{2} \beta^{2}}{d_{0}^{2}}\left[2 d_{0}-4\right] .
$$

Thus, since $d_{0}<0$, we have $\mathcal{M}\left(\xi_{c}\right)<0$. By the intermediate value theorem, it follows that $\mathcal{M}(\xi)=0$ must have two real roots $\xi_{ \pm}$, which satisfy $\beta^{2}<\xi_{-}<\xi_{c}$ and $\xi_{c}<\xi_{+}$. However, since $A>0$ for $\xi=\xi_{-}<\xi_{c}$ and $A<0$ for $\xi=\xi_{+}>\xi_{c}$, only the smaller of the two roots yields a $\tau_{H}>0$ from (3.23a). Therefore, the smaller root $\xi_{-}$gives the Hopf bifurcation, and this root determines $\lambda_{I}$ as $\lambda_{I}=\sqrt{\xi_{-}-\beta^{2}}$.

This completes the proof of (3.23).

For the special case where $d_{0}=0, \mathcal{M}(\xi)=0$ has a unique root in $\xi>\beta^{2}$ given by

$$
\xi=c_{0}+\sqrt{c_{1}}, \quad c_{0}=-\frac{d_{1}^{2}}{8}, \quad c_{1}=\frac{d_{1}^{4}}{64}+\frac{\beta^{2} d_{1}^{2}}{2} .
$$

Then, from (3.27) with $d_{0}=0$, we get

$$
\tau=\frac{d_{1}^{2} \beta}{2}\left(\frac{1}{c_{0}+\sqrt{c_{1}}}\right)^{2}=\frac{d_{1}^{2} \beta}{2\left(c_{0}^{2}-c_{1}\right)}\left(c_{0}^{2}+c_{1}-2 c_{0} \sqrt{c_{1}}\right) .
$$


Since $c_{0}^{2}-c_{1}=-\beta^{2} d_{1}^{2} / 2$, we obtain

$$
\tau=\frac{d_{1}^{2} \beta}{2}\left(\frac{2}{\beta^{2} d_{1}^{2}}\right)^{2}\left[\frac{d_{1}^{4}}{32}+\frac{\beta^{2} d_{1}^{2}}{2}+\frac{d_{1}^{2}}{4} \sqrt{\frac{d_{1}^{4}}{64}+\frac{\beta^{2} d_{1}^{2}}{2}}\right]=\frac{1}{\beta}\left[\frac{d_{1}^{2}}{16 \beta^{2}}+1-\frac{d_{1}}{2 \beta \sqrt{2}} \sqrt{1+\frac{d_{1}^{2}}{32 \beta^{2}}}\right] .
$$

This last expression is equivalent to (3.24a).

Remark 3.12. Recall from Proposition 3.6 that for the case where $d_{1}<0$ and $d_{0}>2$, so that necessarily $\mathcal{G}(0)>2$, we have $N=2$ when both $\tau \ll 1$ and $\tau \gg 1$. However, the behaviour of the eigenvalues for intermediate values of $\tau$ is unclear, since Proposition 3.7 only proves that either $N=0$ or $N=2$. This leaves open the possibility that at some intermediate range of values of $\tau$ a pair of complex conjugate eigenvalues can cross into the negative half-plane $\operatorname{Re}(\lambda)<0$, and then only re-appear in $\operatorname{Re}(\lambda)>0$ at some sufficiently large value of $\tau$. Our last result below, given in Proposition 3.13, rules out this possibility when $d_{0}>d_{0 c}>2$, where $d_{0 c}$ is some explicit threshold. It also gives a range of values of $d_{0}>2$ that guarantee that a stabilization of the spike will occur for some intermediate range of value of $\tau$.

Proposition 3.13. Suppose that $d_{1}<0$ and that $d_{0}>d_{0 c}>2$, where $d_{0 c}$ is given by

$$
d_{0 c}=\min \left(d_{0 c 1}, d_{0 c 2}\right), \quad d_{0 c 1} \equiv \frac{d_{1}}{2 \beta}+\sqrt{8+\frac{d_{1}^{2}}{2 \beta^{2}}}, \quad d_{0 c 2} \equiv \sqrt{4+\frac{d_{1}^{2}}{4 \beta^{2}}} .
$$

Then, for any $\tau>0$, there are exactly two eigenvalues in $\operatorname{Re}(\lambda)>0$. Hence, the NLEP of Proposition 3.2 does not admit a Hopf bifurcation, and a one-spike solution to (1.2) is spectrally unstable for all $\tau>0$. Moreover, on the range

$$
2<d_{0}<d_{0 m} \equiv \min \left(d_{0 c 1}, d_{0 H}\right), \quad d_{0 H} \equiv \frac{d_{1}}{2 \beta}+\sqrt{4+\frac{3 d_{1}^{2}}{4 \beta^{2}}},
$$

there are exactly two Hopf bifurcation values of $\tau$, labelled by $\tau_{H-}$ and $\tau_{H+}$, with $\tau_{H-}<\tau_{H+}$. The spike is spectrally unstable when $0<\tau<\tau_{H-}$ and when $\tau>\tau_{H+}$, but is stable on the intermediate range $\tau_{H-}<\tau<\tau_{H+}$. Finally, if $d_{0 H}<d_{0}<d_{0 c 1}$ then the quadratic $\mathcal{M}(\xi)=0$ has two real roots $\xi_{ \pm}$, but they both satisfy $\xi_{ \pm}<0$, and so are not in the region $\xi>\beta^{2}$. Hence in this range of $d_{0}$ there are no Hopf bifurcations.

Proof. We first show from the winding number criterion leading to (3.16) that $N=2$ when $d_{0}>d_{0 c 2}$, where $d_{0 c 2}$ is defined in (3.31). To obtain this result, it is sufficient to show that $\mathcal{F}_{I}>0$ at the root of $\mathcal{F}_{R}=0$ when $d_{0}>d_{0 c 2}$. We first set $\mathcal{F}_{R}=0$ in (3.17a) to get

$$
\sqrt{2}[\sqrt{a}+1]^{1 / 2}=d_{0}-\frac{d_{1} \beta}{\xi}, \quad \text { where } \quad a \equiv 1+\tau^{2} \lambda_{I}^{2} \quad \text { and } \quad \xi \equiv \beta^{2}+\lambda_{I}^{2} .
$$

From (3.33) we calculate

$$
\sqrt{a}=\frac{A^{2}}{2}-1, \quad \text { where } \quad A \equiv d_{0}-\frac{d_{1} \beta}{\xi} .
$$

We can then evaluate $\mathcal{F}_{I}$ in $\left(3.17\right.$ a) at this root of $\mathcal{F}_{R}$ to get

$$
\mathcal{F}_{I}=\sqrt{2}[\sqrt{a}-1]^{1 / 2}+\frac{d_{1} \lambda_{I}}{\beta^{2}+\lambda_{I}^{2}}=\mathcal{H}(A)+\frac{d_{1} \lambda_{I}}{\beta^{2}+\lambda_{I}^{2}}, \quad \text { where } \quad \mathcal{H}(A) \equiv\left(A^{2}-4\right)^{1 / 2} .
$$

We note that $\mathcal{H}(2)=0$ and $\mathcal{H}^{\prime}(A)>0$ for $A>2$. In addition, for $d_{0}>2$ and $d_{1}<0$, we have $A>d_{0}>2$ for all $\xi>\beta^{2}$. Therefore, since $d_{1}<0$, we calculate a lower bound for $\mathcal{F}_{I}$ as

$$
\mathcal{F}_{I} \geq \mathcal{H}\left(d_{0}\right)+d_{1} \max _{\lambda_{I}>0} \frac{\lambda_{I}}{\beta^{2}+\lambda_{I}^{2}}=\left(d_{0}^{2}-4\right)^{1 / 2}+\frac{d_{1}}{2 \beta} .
$$


We conclude that $\mathcal{F}_{I}>0$ at the root of $\mathcal{F}_{R}=0$, when $d_{0}$ is sufficiently large, so that $\left(d_{0}^{2}-4\right)^{1 / 2}>$ $-d_{1} /(2 \beta)$. This yields that $N=2$ for any $\tau>0$ when $d_{0}>d_{0 c 2}$.

To obtain the second result that $N=2$ when $d_{0}>d_{0 c 1}$, where $d_{0 c 1}$ is defined in (3.31), we proceed by examining the roots of the quadratic polynomial $\mathcal{M}(\xi)=0$ given in $(3.23 \mathrm{~b})$ on the range $\xi>\beta^{2}$ when $d_{0}>2$. When $d_{0}>2$, we have $\mathcal{M} \rightarrow+\infty$ as $\xi \rightarrow \infty$ and $\mathcal{M}\left(\beta^{2}\right)>0$ from (3.29b). Therefore, a sufficient condition for $\mathcal{M}>0$ on $\xi>\beta^{2}$ is that the discriminant associated with the polynomial is negative, i.e. that

$$
\left(d_{1}^{2}+2 \beta d_{1} d_{2}\right)^{2}-8 \beta^{2} d_{1}^{2}\left(d_{0}^{2}-4\right)<0 .
$$

A simple calculation from this inequality yields that $d_{0}>d_{0 c 1}$ where $d_{0 c 1}$ is defined in (3.31).

The last step of the proof is to determine sufficient conditions for which $\mathcal{M}(\xi)=0$ has two roots in $\xi>\beta^{2}$. Since $d_{0}>0$ and $d_{1}<0$, then $A>0$ in (3.23a), and so these roots determine two Hopf bifurcation points $\tau_{H-}$ and $\tau_{H+}$ with $\tau_{H-}<\tau_{H+}$. A necessary and sufficient condition for this is that the discriminant associated with the quadratic is positive, and that the minimum point of $\mathcal{M}(\xi)$ versus $\xi$ occurs in the region $\xi>\beta^{2}$. This occurs when both

$$
\left(d_{1}^{2}+2 \beta d_{1} d_{2}\right)^{2}-8 \beta^{2} d_{1}^{2}\left(d_{0}^{2}-4\right)>0 \quad \text { and } \quad d_{1}^{2}+2 \beta d_{0} d_{1}>2 \beta^{2}\left(d_{0}^{2}-4\right)
$$

hold simultaneously. A simple manipulation of these inequalities yields $2<d_{0}<d_{0 m} \equiv \min \left(d_{0 H}, d_{0 c 1}\right)$, where $d_{0 H}$ is defined in (3.32).

Next, we illustrate Proposition 3.13 graphically by plotting the regions in the parameter space $d_{0}>2$ versus $t \equiv-d_{1} /(2 \beta)$ where we can guarantee the number of Hopf bifurcation points. In terms of $t$ we have from (3.31) and (3.32) that

$$
d_{0 c 1}=t+\sqrt{8+2 t^{2}}, \quad d_{0 c 2}=\sqrt{4+t^{2}}, \quad d_{0 H}=t+\sqrt{4+3 t^{2}} .
$$

We first consider the range $t<-2$. On this range, it is readily shown that $d_{0 H}>d_{0 c 1}$ and $d_{0 c 2}>d_{0 c 1}$. Therefore, when $t<-2$, we conclude that if $d_{0}>d_{0 c 1}$ there are no Hopf bifurcations, while for $2<d_{0}<$ $d_{0 c 1}$ we have two Hopf bifurcation points. The curve $d_{0}=d_{0 c 1}$ for $t<-2$ is where $\mathcal{M}(\xi)=0$ has a double root in the region $\xi>\beta^{2}$. Next, consider the range $-2<t<0$. A simple calculation shows that $d_{0 c 1}<$ $d_{0 c 2}$ for $-2<t<t_{s}$, whereas $d_{0 c 2}<d_{0 c 1}$ on $t_{s}<t<0$. Here $t_{s}=-\sqrt{2(\sqrt{2}-1)} \approx-0.910$. In addition, we readily calculate that $d_{0 H}<2$ on $-2<t<0$. Thus, on $-2<t<0$, where $d_{0 H}<2<d_{0}<d_{0 c 1}$ it follows that $\mathcal{M}(\xi)=0$ has two real roots, with neither in the region $\xi>\beta^{2}$. For $d_{0}>\min \left(d_{c 1}, d_{c 2}\right)$, there are no Hopf bifurcations. We conclude that there are no Hopf bifurcations possible in the region where $-2<t<0$. These considerations yield the phase diagram of Fig. 8 characterizing Hopf bifurcations in the entire parameter plane $d_{0}>2$ versus $t \equiv-d_{1} /(2 \beta)<0$.

Remark 3.14. The parameter plane shown in Fig. 8 predicts either zero or two Hopf bifurcation points in the entire parameter plane where $d_{0}>2$ and $d_{1}<0$. To use this result for arbitrary kinetics $f$ and $g$, we need only solve the nonlinear algebraic problem (3.4) for $U_{0}$, and then compute $d_{0}$ and $d_{1}$ in terms of $U_{0}$ from (2.15b). If $d_{0}>2$ and $d_{1}<0$, this determines a point in the parameter space of Fig. 8 .

\subsection{Two Examples of the Theory}

Since the instability results for eigenvalues on the positive real axis for the infinite line problem are essentially the same as for the shadow system studied in $\S 2.2$, we will only illustrate the Hopf bifurcation results contained in Propositions 3.11 and 3.13.

GM Model: Consider a one-spike solution on the infinite line for the GM model where $g(u)=u^{-q}$, $f(u)=u^{-s}$ and $u_{b}=0$. Then, $d_{0}=-2 s, d_{1}=-q r^{2}$, and $\beta=r^{2}-2 r$, as were given in (2.23). 


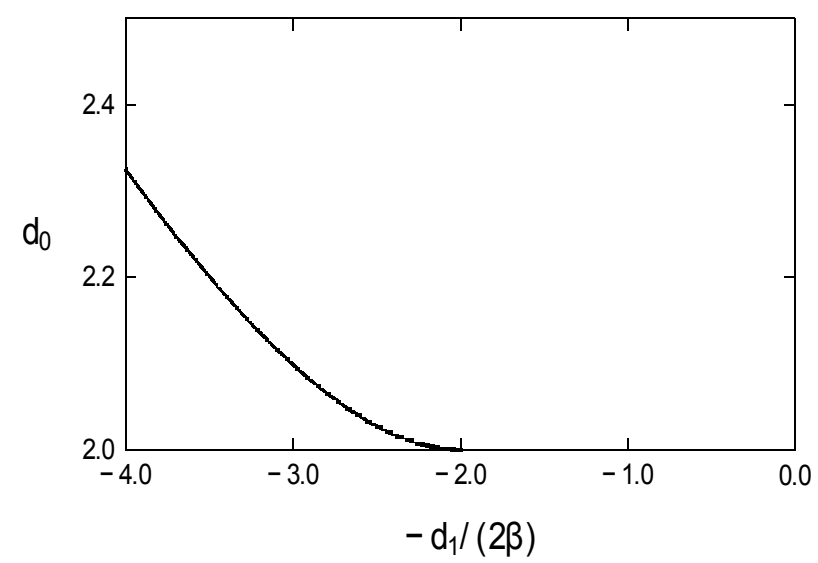

FiguRE 8 . Plot of the region in the $d_{0}>2$ versus $t \equiv-d_{1} /(2 \beta)$ parameter illustrating Proposition 3.13. For $t<-2$, there are no Hopf bifurcation points above the heavy solid curve, and two Hopf bifurcation points below the heavy solid curve. The heavy solid curve is $d_{0 c 1}=t+\sqrt{8+2 t^{2}}$. For $-2<t<0$, there are no Hopf bifurcation points for any $d_{0}>2$. It is only the region $2<d_{0}<d_{0 c 1}$ and $t<-2$ where a one-spike solution will be stable only on some intermediate range $0<\tau_{H-}<\tau<\tau_{H+}<\infty$.

For the special case $s=0$, for which $d_{0}=0$, then $\tau$ is given by (3.24) where we identify $c$ as

$$
c=\frac{q r}{2 \sqrt{2}(r-2)} .
$$

In particular, for the GM exponent set $(p, q, r, s)=(3,2,3,0)$, we calculate from (3.24) and (3.37) that

$$
\tau_{H}=\frac{1}{12}[13+3 \sqrt{17}] \approx 2.114, \quad \lambda_{I}=3 \sqrt{1-\frac{2}{3 \tau_{H}}} \approx 2.482
$$

More generally, fixing $p=r=3$ and $s=0$, a plot of $\tau_{H}$ versus $q$ is shown by the heavy solid curve in Fig. 9.

Next, consider the GM exponent set $(p, q, r, s)=(3,2,3,1)$. For this set, the Hopf bifurcation threshold for a one-spike solution on a finite domain was computed using the full numerical approach of [37], and is shown in Fig. 4 of [37]. The $y$-intercept of this plot of [37] is the corresponding result for our infinite-line problem. For the exponent set $(p, q, r, s)=(3,2,3,1)$, for which $d_{0}=-2$, the quadratic polynomial $\mathcal{M}(\xi)=0$ in $(3.23 \mathrm{~b})$ degenerates to a straight line, and we calculate that $\xi=2 \beta^{2} d_{1} /\left(d_{1}-4 \beta\right)=54 / 5$. We calculate from (3.23) that $\tau_{H}=2.5$ and $\lambda_{I}=3 / \sqrt{5} \approx 1.34$ in agreement with the results given on the $y$-intercept of Fig. 4 of [37]. More generally, the dotted curve of Fig. 9 is a plot of $\tau_{H}$ versus $q$ for the exponent set $(p, q, r, s)=(3, q, 3,1)$, as obtained from (3.23).

To illustrate the use of Fig. 8, we consider the GM model with exponent set $(p, q, r, s)$ with $p=2 r-3$, $r>2, q>0$ and $s<-1$. Since $d_{0}=-2 s$ and $d_{1}=-q r^{2}$, we conclude that $d_{0}>2$ and $d_{1}<0$. If $-2<d_{1} /(2 \beta)<0$, then we predict that there are no Hopf bifurcations for any $\tau>0$, and the spike is always unstable. This inequality holds when $q<4(r-2) / r$. Next, suppose that $q>4(r-2) / r$. Then, we obtain that there are no Hopf Bifurcations when

$$
s<-\frac{q r}{4(r-2)}-\frac{1}{2} \sqrt{8+\frac{q^{2} r^{2}}{2(r-2)^{2}}},
$$




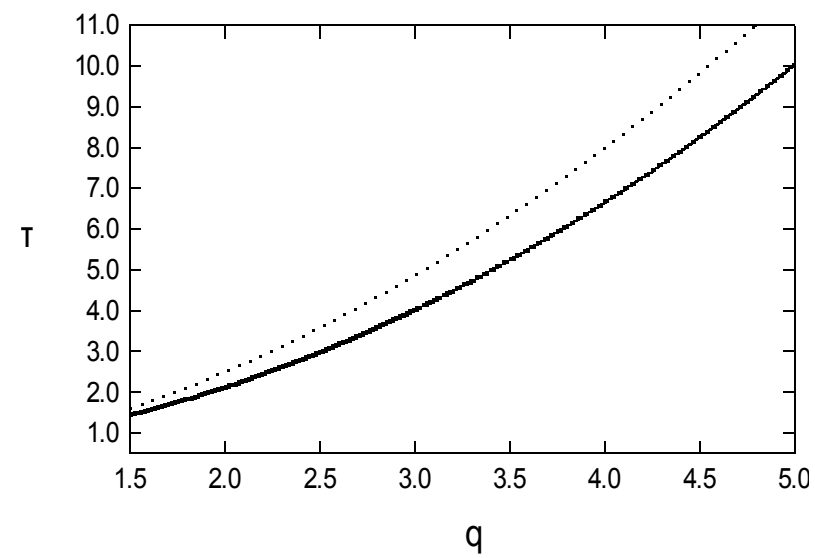

Figure 9. Plot of $\tau_{H}$ versus $q$ for the GM model with $p=r=3$ and for $s=1$ (dotted curve) and for $s=0$ (heavy solid curve). For $s=0$ and $s=1, \tau$ is calculated from (3.24) and (3.23), respectively

and that there are two Hopf bifurcations when

$$
-\frac{q r}{4(r-2)}-\frac{1}{2} \sqrt{8+\frac{q^{2} r^{2}}{2(r-2)^{2}}}<s<-1 .
$$

For the range of $s$ in $(3.38)$ and for $q>4(r-2) / r$, a one-spike solution will be stable only on some intermediate range of $\tau$.

Generalized GS Model: Next, we consider the generalized GS model (1.2) for which

$$
p=r=3, \quad f(u)=-u, \quad g(u)=\mathcal{A} u, \quad u_{b}=1 .
$$

To construct the one-spike equilibrium solution on the infinite line we must calculate $U_{0}$ from (3.4). In this way, we find that $U_{0}$, on $0<U_{0}<1$, again satisfies (2.31) when $\mathcal{A}>\mathcal{A}_{m}$, where $\mathcal{A}_{m}$ is defined in (2.32). The graph of $U_{0}$ versus $\mathcal{A}$ has a saddle-node bifurcation structure, with two solutions $U_{0 \pm}$ for $U_{0}$ on $0<U_{0-}<1 / 3<U_{0+}<1$ when $\mathcal{A}>\mathcal{A}_{m}$ (see Fig. 4).

For the stability analysis, we identify $\beta=r^{2}-3 r=3$, and calculate $d_{0}$ and $d_{1}$ from (2.15b) as

$$
d_{0}=-\frac{2\left(1-U_{0}\right)}{U_{0}}<0, \quad d_{1}=-\frac{9\left(1-U_{0}\right)}{U_{0}}<0, \quad \mathcal{G}(0)=\frac{\left(1-U_{0}\right)}{U_{0}} .
$$

Since $\mathcal{G}(0)=\left(1-U_{0}\right) / U_{0}<2$ when $U_{0}>1 / 3$, we conclude from (I) of Proposition 3.5 that on the entire upper branch $1 / 3<U_{0}<1$ the spike is unstable due to a positive real eigenvalue for any $\tau>0$.

On the lower branch, where $0<U_{0}<1 / 3$, a one-spike solution undergoes a Hopf bifurcation at some unique $\tau=\tau_{H}>0$. Since $d_{0}=-2\left(1-U_{0}\right) / U_{0}$ and $0<U_{0}<1 / 3$, we conclude that $d_{0}<-4, d_{1}<0$, and $\mathcal{G}(0)>2$ on this branch. Consequently, Case III in the proof of Proposition 3.11 applies, and $\mathcal{M}(\xi)=0$ in (3.23b) has two positive roots in $\xi>\beta^{2}$, with the smaller root corresponding to the Hopf bifurcation point. By using (3.40) in (3.23), and calculating the smallest root of $\mathcal{M}(\xi)=0$, we obtain for each $U_{0}$ in $0<U_{0}<1 / 3$ that

$$
\tau_{H}=\frac{81\left(1-U_{0}\right)^{2}}{2 U_{0}^{2}}\left(\frac{3}{\xi^{2}}-\frac{2}{9 \xi}\right), \quad \xi=\frac{\left(1-U_{0}\right)^{2}}{2\left(1-2 U_{0}\right)}\left[\frac{189}{4}-\sqrt{\left(\frac{189}{4}\right)^{2}-1458\left(\frac{\left(1-2 U_{0}\right)}{\left(1-U_{0}\right)^{2}}\right)}\right] .
$$

In Fig. 10 we plot $\tau_{H}$ versus $U_{0}$ on $0<U_{0}<1 / 3$ and versus $\mathcal{A}$ where $\mathcal{A}>\mathcal{A}_{m}$. At the saddle node bifurcation point where $U_{0}=1 / 3$ and $\mathcal{A}=\mathcal{A}_{m} \approx 3.218$, we calculate $\tau_{H}=2$ from (3.41). 

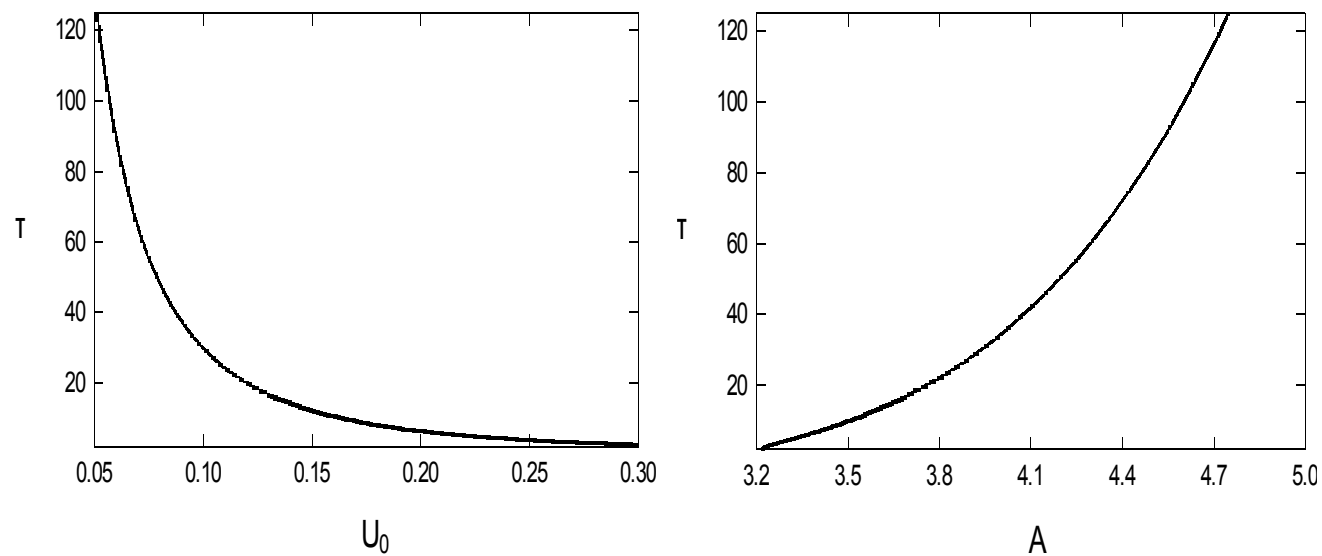

FiguRE 10. Plot of the Hopf bifurcation threshold $\tau_{H}$ for the generalized GS model versus $U_{0}$ (left panel) and versus $\mathcal{A}$ (right panel), corresponding to the lower branch $0<U_{0}<1 / 3$ of Fig. 4. Here $\tau_{H}$ is given in (3.41),

\subsection{NLEP Stability Theory with Sub-Diffusion}

Finally, we remark that completely analogous results to those in $\S 2.4$ for the sub-diffusive shadow problem hold for the sub-diffusive infinite-line problem (1.3).

More specifically, to analyze the stability of a one-spike solution for (1.3) we simply replace $\lambda$ in (3.13) with $\lambda^{(0)^{\gamma}}=\lambda$. We recall from $\S 2.4$ that an instability for the sub-diffusive problem can only occur when the roots of (3.13) are in the wedge of instability defined by (see Fig. 7)

$$
-\frac{\pi \gamma}{2} \leqslant \arg (\lambda) \leqslant \frac{\pi \gamma}{2}, \quad|\lambda| \gg \mathcal{O}(\varepsilon),
$$

where the anomaly exponent $\gamma$ is on the range $0<\gamma<1$.

As such, we conclude that if the regular NLEP admits a positive real eigenvalue $\lambda^{*}>0$, then this eigenvalue must lie in the wedge of instability for the sub-diffusive NLEP for any $\gamma$ in $0<\gamma<1$. Such an eigenvalue $\lambda^{(0)}=\left(\lambda^{*}\right)^{1 / \gamma}>0$ yields an exponentially growing perturbation (to leading order) to the one-spike equilibrium solution of (1.3). Consequently, the instability results of Propositions 3.5 and 3.6, which result from a positive real eigenvalue of the regular diffusion NLEP, still hold for the sub-diffusion problem for all $\gamma$ in $0<\gamma<1$. We summarize this result as follows:

Proposition 3.15. Suppose that either

(I) $\quad d_{1}<0$ and $\mathcal{G}(0)<2 ; \quad$ or $\quad(I I) \quad d_{1}>0$ and $\mathcal{G}(0)>2 ; \quad$ or $(I I I) \quad d_{1}>0$ and $d_{0}<2$.

Then, for any $\tau>0$, there is an exponentially growing perturbation to the one-spike equilibrium solution of the sub-diffusive system (1.3). Similarly, when $d_{1}<0, \mathcal{G}(0)>2$, and $d_{0}<2$, the one-spike equilibrium solution of (1.3) is unstable when $\tau>0$ is sufficiently large.

Next, we consider Hopf bifurcations associated with the sub-diffusive RD system (1.3) under the conditions that $d_{1}<0, d_{0}<2$ and $\mathcal{G}(0)>2$. For this parameter range, Proposition 3.11 proves that the regular diffusion problem undergoes a Hopf bifurcation at a unique value $\tau=\tau_{H}>0$, and by Proposition 3.6 there are two positive real eigenvalues for the regular NLEP when $\tau \gg 1$. More specifically, there are two positive real roots of (3.13) for $\tau>\tau_{t}$, where $\tau_{t}>0$ is the unique value of $\tau$ at which $2 \sqrt{1+\tau \lambda}$ and $\mathcal{G}(\lambda)$ in (3.13) intersect tangentially at some $\lambda=\lambda_{t}>0$. The key observation is that an unstable eigenvalue of the regular NLEP only generates an instability for the sub-diffusive NLEP when it lies 

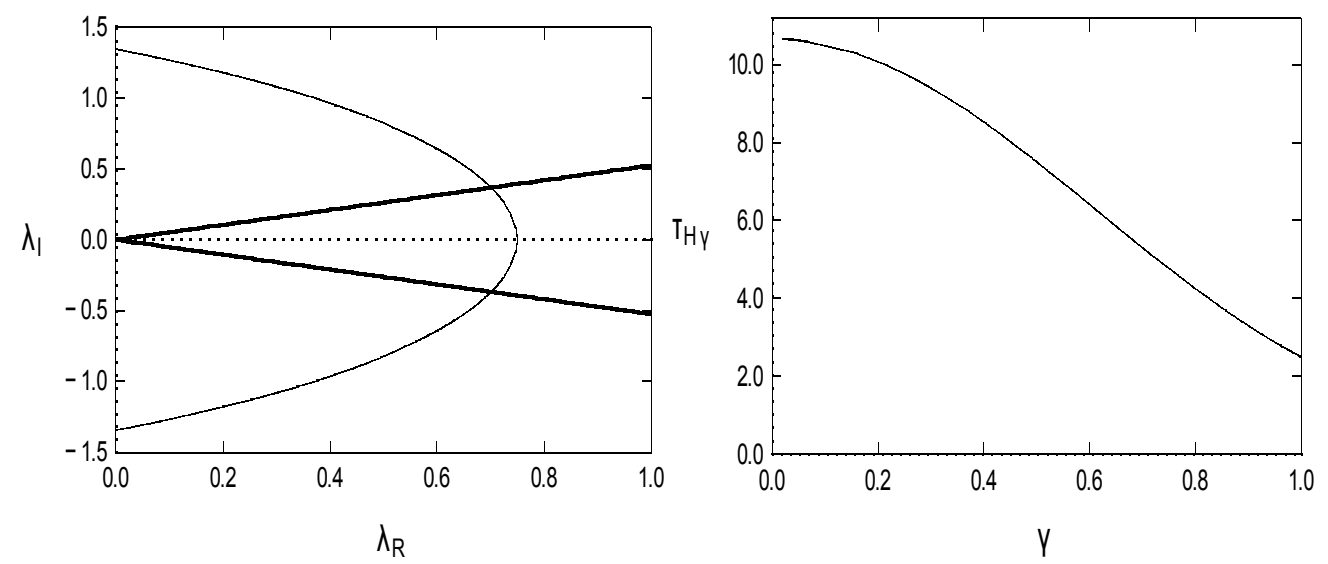

FiguRE 11. Hopf bifurcation for the GM model corresponding to setting $p=r=3$, $g(u)=u^{-2}, f(u)=u^{-1}$ and $u_{b}=0$ in (1.2) and (1.3). Left panel: the plot of the path $\lambda(\tau)$ (solid curve) of the complex conjugate eigenvalue pair for the NLEP with regular diffusion on the range $\tau_{H}<\tau<\tau_{t}$. The wedge of instability for the sub-diffusive problem when $\gamma=1 / 3$ lies between the two heavy solid lines. For values of $\tau$ for which $\lambda(\tau)$ is inside this wedge, the one-spike solution for the sub-diffusive GM model has an oscillatory instability. Right panel: the Hopf bifurcation threshold $\tau_{H \gamma}$ versus $\gamma$ corresponding to when the path $\lambda(\tau)$ intersects the edge of the wedge of instability.

within the wedge of instability (3.42). This wedge of instability becomes narrower as $\gamma$ decreases, and concentrates on the positive real axis in the $\lambda$-plane as $\gamma \rightarrow 0$. The anomaly-dependent Hopf bifurcation threshold, labelled by $\tau_{H \gamma}$, is computed numerically from the implicit condition that

$$
\arg \lambda=\frac{\pi \gamma}{2},
$$

which involves the eigenvalue path $\lambda=\lambda(\tau)$ of the roots of (3.13) with $\operatorname{Re}(\lambda)>0$ and $\operatorname{Im}(\lambda)>0$. This condition (3.43) corresponds to when this eigenvalue path intersects the edge of the wedge of instability shown in Fig. 7. In this sense, we conclude that the sub-diffusive RD system (1.3) undergoes an oscillatory instability at a larger value of $\tau$ than for the case of regular diffusion. We summarize this result as follows:

Proposition 3.16. Suppose that $d_{0}<2, d_{1}<0$ and $\mathcal{G}(0)>2$. Then, for all $\gamma$ in $0<\gamma<1$, the Hopf bifurcation threshold $\tau_{H \gamma}$ for the sub-diffusive NLEP satisfies $\tau_{H}<\tau_{H \gamma}<\tau_{t}$. Here $\tau_{H}$ is the Hopf bifurcation threshold for the regular diffusion case, as given by Proposition 3.11, and $\tau_{t}$ is the value of $\tau$, where the complex conjugate eigenvalue path for the regular NLEP merge onto the positive real axis.

In addition, suppose that the eigenvalue path for the regular NLEP in the first quadrant $\operatorname{Im}(\lambda)>0$ and $\operatorname{Re}(\lambda)>0$ is monotonic in the sense that $\operatorname{Re}(\lambda)$ increases monotonically while $\operatorname{Im}(\lambda)$ decreases monotonically as $\tau$ increases. Then, the Hopf bifurcation threshold $\tau_{H \gamma}$ for the sub-diffusive NLEP increases as $\gamma$ decreases.

In Fig. 11 we illustrate Proposition 3.16 for the GM model corresponding to setting $p=r=3$, $g(u)=u^{-2}, f(u)=u^{-1}$ and $u_{b}=0$ in (1.2) and (1.3). For this example $d_{0}=-2, d_{1}=-18$, and $\mathcal{G}(0)=4>2$. In the left panel of Fig. 11 we plot the path $\lambda(\tau)$ of the complex conjugate eigenvalue pair for the NLEP with regular diffusion on the range $\tau_{H}<\tau<\tau_{t}$, as computed from (3.13). We calculate $\tau_{t} \approx 10.68$ and from Proposition 3.11 we get $\tau_{H}=2.5$. In Fig. 11, we also plot the wedge of instability for the sub-diffusive problem when $\gamma=1 / 3$. For this example, the eigenvalue path of the regular NLEP is monotonic in the sense described above. Therefore, $\tau_{H \gamma}$ increases as $\gamma$ decreases. This Hopf bifurcation threshold of the sub-diffusive problem is shown in the right panel of Fig. 11. 
We remark that the analysis for the case $d_{0}>2$ and $d_{1}<0$ is more intricate. Recall that the Hopf bifurcation result for the regular NLEP for this parameter range was given in Proposition 3.13. In particular, when $d_{0}$ satisfies (3.32), the regular NLEP has two Hopf bifurcation thresholds $\tau_{H \pm}>0$ with a one-spike solution being stable on the intermediate range $\tau_{H-}<\tau<\tau_{H+}$, and unstable otherwise. However, since an unstable eigenvalue of the regular NLEP only generates an instability for the subdiffusive problem when it lies within the wedge of instability (3.42), it follows for any $\gamma$ in $0<\gamma<1$ that this intermediate stability zone in $\tau$ will be larger for the sub-diffusive problem.

\section{Discussion}

We have studied the existence and linearized stability of a one-spike equilibrium solution to the class of RD systems (1.2) on the infinite-line. A similar analysis has been done for the corresponding shadow system (2.2). For arbitrary $p \geq 2$ and $r>1$ in (1.2) and (2.2), the problem of the existence of a one-spike solution was reduced to the study of the scalar nonlinear algebraic equation (3.4) and (2.6), respectively. To determine the linearized stability of the one-spike solutions, we used the method of matched asymptotic expansions to derive a nonlocal eigenvalue problem (NLEP). For the special case where $p=2 r-3$ and $r>2$, we showed that the spectrum of this NLEP can be reduced to the study of the roots of a rather simple transcendental equation involving $\tau, r$, and two key parameters $d_{0}$ and $d_{1}$ related to the specific choices of the kinetic functions of the nonlinearities $f(u)$ and $g(u)$ in (1.2) and (2.2).

From an analysis of these transcendental equations by using a winding number approach together with detailed analytical calculations, explicit stability and instability results were obtained in terms of ranges of the two parameters $d_{0}, d_{1}$, and the reaction-time constant $\tau$. Most notably, in certain parameter regimes of $d_{0}$ and $d_{1}$ our theory provides sufficient conditions for the existence of a unique Hopf bifurcation value of $\tau$, as well as a simple analytical formula to calculate this threshold. The theory was illustrated for the GM model and for a generalization of the GS model.

There are several related problems that can be investigated within the simple framework afforded by an explicitly solvable NLEP. The first problem is to characterize analytically the slow dynamics and stability of a two-spike pattern on the infinite line for the regular diffusion problem (1.2) and its subdiffusive counterpart (1.3). For the case $p=2 r-3$ with $r>2$, for which the associated NLEP is explicitly solvable, it should be possible to provide an explicit theory characterizing both competition and oscillatory instabilities of the two-spike pattern. Results in this direction are given in [25]. A second open problem is to investigate delayed bifurcation effects for the stability of pulses due to either the slow drift of the pulse locations or due to slowly varying extrinsic control parameters, such as $\tau$ or the length of the domain. Finally, as mentioned in Remark 2.8, it would be interesting to investigate whether more general non power-law nonlinearities can also lead to an explicitly solvable NLEP. Results in this direction are given in [32].

Acknowledgements. M. J. W. is grateful for the grant support of NSERC.

\section{References}

[1] W. Chen, M. J. Ward. Oscillatory instabilities of multi-spike patterns for the one-dimensional Gray-Scott model. Europ. J. Appl. Math., 20 (2), (2009), pp. 187-214.

[2] W. Chen, M. J. Ward. The stability and dynamics of localized spot patterns in the two-dimensional Gray-Scott model. SIAM J. Appl. Dyn. Sys., 10 (2), (2011), pp. 582-666.

[3] A. Doelman, W. Eckhaus, T. J. Kaper. Slowly modulated two-pulse solutions in the Gray-Scott model $i$ : asymptotic construction and stability. SIAM J. Appl. Math., 61 (3), (2000), pp. 1080-1102.

[4] A. Doelman, W. Eckhaus, T. J. Kaper. Slowly modulated two-pulse solutions in the Gray-Scott model ii: geometric theory, bifurcations, and splitting dynamics. SIAM J. Appl. Math., 61 (6), (2000), pp. 2036-2061.

[5] A. Doelman, R. A. Gardner, T. Kaper. Large stable pulse solutions in reaction-diffusion equations. Indiana U. Math. Journ., 50 (1), (2001), pp. 443-507.

[6] A. Doelman, T. Kaper. Semistrong pulse interactions in a class of coupled reaction-diffusion equations. SIAM J. Appl. Dyn. Sys., 2 (1), (2003), pp. 53-96. 
[7] A. Doelman, T. Kaper, K. Promislow. Nonlinear asymptotic stability of the semi-strong pulse dynamics in a regularized Gierer-Meinhardt model. SIAM J. Math. Anal., 38(6), (2007), pp. 1760-1789.

[8] A. Doelman, H. van der Ploeg. Homoclinic stripe patterns. SIAM J. Appl. Dyn. Sys., 1 (1), (2002), pp. 65-104.

[9] J. Ehrt, J. D. Rademacher, M. Wolfrum. First and second order semi-strong interaction of pulses in the Schnakenberg model. preprint, (2012).

[10] A. A. Golovin, B. J. Matkowsky, V. A. Volpert. Turing pattern formation in the Brusselator model with superdiffusion. SIAM J. Appl. Math., 69 (1), (2008), pp. 251-272.

[11] B. I. Henry, S. L. Wearne. Existence of Turing instabilities in a two-species fractional reaction-diffusion system. SIAM J. Appl. Math., 62 (3), (2002), pp. 870-887.

[12] D. Iron, M. J. Ward. A metastable spike solution for a non-local reaction-diffusion model. SIAM J. Appl. Math., 60 (3), (2000), pp. 778-802.

[13] D. Iron, M. J. Ward, J. Wei. The stability of spike solutions to the one-dimensional Gierer-Meinhardt model. Physica D, 150 (1-2), (2001), pp. 25-62.

[14] D. Iron, M. J. Ward. The dynamics of multi-spike solutions to the one-dimensional Gierer-Meinhardt model. SIAM J. Appl. Math., 62 (6), (2002), pp. 1924-1951.

[15] T. Kolokolnikov, M. J. Ward, J. Wei. The stability of spike equilibria in the one-dimensional Gray-Scott model: the low feed-rate regime. Studies in Appl. Math., 115 (1), (2005), pp. 21-71

[16] T. Koloklonikov, M. J. Ward, J. Wei. Slow translational instabilities of spike patterns in the one-dimensional GrayScott model. Interfaces and Free Boundaries, 8 (2), (2006), pp. 185-222.

[17] T. Kolokolnikov, M. Ward, J. Wei. The stability of steady-state hot-spot patterns for a reaction-diffusion model of urban crime. Disc. Cont. Dyn. Sys Series B., to appear, (2013), (34 pages).

[18] C. S. Lin, W. M. Ni, I. Takagi. Large amplitude stationary solutions to a chemotaxis system. J. Diff. Eq., 72 (1), (1988), pp. 1-27.

[19] R. Metzler, J. Klafter. The random walk's guide to anomalous diffusion: a fractional dynamics approach. Phys. Rep., 339. (2000), pp. 1-77.

[20] C. Muratov, V. V. Osipov. Stability of the static spike autosolitons in the Gray-Scott model. SIAM J. Appl. Math., 62 (5), (2002), pp. 1463-1487.

[21] Y. Nec, V. A. Volpert, A. A. Nepomnyashchy. Front propagation problems with sub-diffusion. Discr. Cont. Dyn. Sys. Series A, 27 (2), (2010), pp. 827-846.

[22] Y. Nec, A. A. Nepomnyashchy. Linear stability of fractional reaction-diffusion systems. Math. Model. Nat. Phenom., 2 (2), (2007), pp. 77-105.

[23] Y. Nec, A. A. Nepomnyashchy. Turing instability in sub-diffusive reaction-diffusion systems. J. Physics A: Math. Theor., 40 (49), (2007), pp. 14687-14702.

[24] Y. Nec, M. J. Ward. The dynamics and stability of spike-type solutions to the Gierer-Meinhardt model with subdiffusion. Physica D, 241 (9), (2012), pp. 947-963.

[25] Y. Nec, M. J. Ward. The stability and slow dynamics of two-spike patterns for a class of reaction-diffusion system. submitted, (2013), (28 pages)

[26] K. B. Oldham, J. Spanier. The fractional calculus. Academic Press, New York, 1974.

[27] I. Podlubny. Fractional differential equations. Academic Press, San Diego, 1999.

[28] J. D. Rademacher. First and second order semi-strong interface interaction in reaction-diffusion systems. SIAM J. App. Dyn. Syst., (2012), to appear.

[29] R. K. Saxena, A. M. Mathai, H. J. Haubold, Fractional reaction-diffusion equations. Astrophys. Space Sci., 305 (3), (2006), pp. 289-296.

[30] W. Sun, T. Tang, M. J. Ward, J. Wei. Numerical challenges for resolving spike dynamics for two reaction-diffusion systems. Studies in Appl. Math., 111 (1), (2003), pp. 41-84.

[31] W. Sun, M. J. Ward, R. Russell. The slow dynamics of two-spike solutions for the Gray-Scott and Gierer-Meinhardt systems: competition and oscillatory instabilities. SIAM J. App. Dyn. Sys., 4 (4), (2005), pp. $904-953$.

[32] W. H. Tse, M. J. Ward. On explicitly solvable nonlocal eigenvalue problems and the stability of localized pulses. to be submitted, Applied Math Letters, (2013).

[33] J. C. Tzou, A. Bayliss, B. J. Matkowsky, V. A. Volpert, Interaction of Turing and Hopf models in the superdiffusive Brusselator model near a codimension two bifurcation point. Math. Model. Nat. Phenom. 6 (1), (2011), pp. 87-118.

[34] J. C. Tzou, A. Bayliss, B. J. Matkowsky, V. A. Volpert, Stationary and slowly moving localized pulses in a singularly perturbed Brusselator model. Europ. J. Appl. Math., 22 (5), (2011), pp. 423-453.

[35] J. C. Tzou, Y. Nec, M. J. Ward, The Stability of Localized Spikes for the 1-D Brusselator Reaction-Diffusion Model. Europ. J. Appl. Math., (2012), under review.

[36] H. van der Ploeg, A. Doelman. Stability of spatially periodic pulse patterns in a class of singularly perturbed reactiondiffusion equations. Indiana U. Math. J., 54 (5), (2005), pp. 1219-1301.

[37] M. J. Ward, J. Wei. Hopf bifurcations and oscillatory instabilities of spike solutions for the one-dimensional GiererMeinhardt model. J. Nonlinear Science, 13 (2), (2003), pp. 209-264.

[38] M. J. Ward, J. Wei. Hopf bifurcations of spike solutions for the shadow Gierer-Meinhardt model. Europ. J. Appl. Math., 14 (6), (2003), pp. 677-711.

[39] J. Wei. On single interior spike solutions for the Gierer-Meinhardt system: uniqueness and stability estimates. Europ. J. Appl. Math., 10 (4), (1999), pp. 353-378. 
[40] J. Wei. Existence and stability of spikes for the Gierer-Meinhardt system. book chapter in Handbook of Differential Equations, Stationary Partial Differential Equations. Vol. 5 (M. Chipot ed.), Elsevier, (2008), pp. 489-581.

[41] M. Wolfrum, J. Ehrt. Slow motion of quasi-stationary multi-pulse solutions by semistrong interaction in reactiondiffusion systems. WIAS Preprint 1233 (2007). 